\title{
DGE-seq analysis of MUR3-related Arabidopsis mutants provides insight into how dysfunctional xyloglucan affects cell elongation
}

Zongchang $\mathrm{Xu}^{\mathrm{a}, \mathrm{b}}$, Meng Wang ${ }^{\mathrm{a}, \mathrm{b}}$, Dachuan Shi ${ }^{\mathrm{a}}$, Gongke Zhou ${ }^{\mathrm{c}}$, Tiantian Niu ${ }^{\mathrm{a}}$, Michael G. Hahn $^{\mathrm{d}, \mathrm{e}}$, Malcolm A. O'Neill ${ }^{\mathrm{d}}$, Yingzhen Kong ${ }^{\mathrm{a}, *}$

${ }^{\text {a }}$ Key Laboratory for Tobacco Gene Resources, Tobacco Research Institute, Chinese Academy of Agricultural Sciences, Qingdao 266101, P.R. China

${ }^{\mathrm{b}}$ Graduate School of Chinese Academy of Agricultural Science, Beijing 100081, China

${ }^{\mathrm{c}}$ Qingdao Engineering Research Center of Biomass Resources and Environment, Qingdao Institute of Bioenergy and Bioprocess Technology, Chinese Academy of Sciences, Qingdao 266101, P.R. China

${ }^{\mathrm{d}}$ Complex Carbohydrate Research Center, University of Georgia, Athens, Georgia 30602-4712 USA

e Department of Plant Biology, University of Georgia, Athens, Georgia 30602-4712 USA

Email addresses:

ZX:xuzc1110@163.com

MW: menglihua@yeah.net

DS: $\underline{\text { shidc@qibebt.ac.cn }}$

GZ: zhougk@qibebt.ac.cn

TN: $\underline{\operatorname{tian} 15288861055 @ 163 . c o m}$

M.G.H: hahn@ccrc.uga.edu

M.A.O: $\underline{\text { mao@ccrc.uga.edu }}$

YK: kongyingzhen@ caas.cn

* Corresponding author; Yingzhen Kong

Phone: +86-532-66715890

Email: kongyingzhen@caas.cn 
Abstract: Our previous study of the Arabidopsis mur3-3 mutant and mutant plants in which the mur3-3 phenotypes are suppressed (xxt2mur3-3, xxt5mur3-3, xxt1xxt2mur3-3 and 35Spro:XLT2:mur3-3) showed that hypocotyl cell elongation is decreased in plants that synthesize galactose-deficient xyloglucan. To obtain genome-wide insight into the transcriptome changes and regulatory networks that may be involved in this decreased elongation, we performed digital gene expression analyses of the etiolated hypocotyls of wild type (WT), mur3-3 and the four suppressor lines. Numerous differentially expressed genes (DEGs) were detected in comparisons between WT and mur3-3 (1423), xxt2mur3-3 and mur3-3 (675), xxt5mur3-3 and mur3-3 (1272), xxt1xxt2mur3-3 and mur3-3 (1197) and 35Spro:XLT2:mur3-3 vs mur3-3 (121). 550 overlapped DEGs were detected among WT vs mur3-3, xxt2mur3-3 vs mur3-3, xxt5mur3-3 vs mur3-3, and $x x t 1 x x t 2 m u r 3-3$ vs mur3-3 comparisons. These DEGs include 46 cell wall-related genes, 24 transcription factors, 6 hormone-related genes, 9 protein kinase genes and 9 aquaporin genes. The expression of all of the 550 overlapped genes is restored to near wild-type levels in the four mur3-3 suppressor lines. qRT-PCR of fifteen of these 550 genes showed that their expression levels are consistent with the digital gene expression data. Overexpression of some of these genes (XTH4, XTH30, PME3, EXPA11, MYB88, ROT3, AT5G37790, WAG2 and TIP2;3) that are down-regulated in mur3-3 partially rescued the short hypocotyl phenotype but not the aerial phenotype of mur3-3, indicating that different mechanisms exist between hypocotyl cell elongation and leaf cell elongation.

${ }^{1}$ Keywords: Arabidopsis; MUR3; xyloglucan; cell elongation; hypocotyl; DGE-seq

\section{Introduction}

Xyloglucan is a hemicellulosic polysaccharide present in the primary cell walls of all land plants studied to date $[1,2]$. Arabidopsis and numerous other flowering plants synthesize a XXXG-type xyloglucan $[3,4]$ in which two of the $\alpha$-D-xylosyl residues (X side chain) attached to the $\beta$-glucan backbone are often substituted at O-2 with a $\beta$-D-Gal $p$ residue (L side chain: XLXG, XXLG and XLLG). The Gal residue of the $\mathbf{L}$ side chain adjacent to the unsubstituted $\mathbf{G}$ is often substituted at O-2 with a $\alpha$-L-Fucp residue (F side chain: XXFG and XLFG) [4].

The Arabidopsis glycosyltransferases (GTs) involved in xyloglucan side chain biosynthesis have been identified [5-10]. These GTs include two XYLOGLUCAN XYLOSYLTRANSFERASES (XXT1 and XXT2) that add xylosyl residues to the glucan backbone [9], XXT5 may also participate in this process but is not essential [10]. XYLOGLUCAN L-SIDE CHAIN GALACTOSYLTRANSFERASE2 (XLT2)

1 Abbreviations: DGE-seq, digital gene expression sequencing; WT, wild type; GO, Gene Ontology; DEGs, differentially expressed genes; GTs, glycosyltransferases; RPKM, reads per kilobases per million reads; KEGG, kyoto encyclopedia of genes and genomes; TFs, transcription factors; BR, brassinosteroids; WGCNA, weighted gene co-expression network analysis. 
adds Gal to the middle Xyl (L side chain) [5], whereas MURUS3 (MUR3) adds Gal to the Xyl adjacent to the unbranched Glc (L side chain) [6]. FUCOSYLTRANSFERASE1 (FUT1) adds a Fuc residue to the MUR3 Gal (F side chain) [7]. The XLFG, XXXG and XXFG subunits account for 43\%, $25 \%$ and $24 \%$, respectively of Arabidopsis xyloglucan [11].

Arabidopsis plants carrying mutations that affect individual GTs have altered xyloglucan structure or content [5, 8, 12-15]. The amounts of Fuc substitution are reduced by at least $98 \%$ in the futl mutant, yet the plants grow normally and the strength of their walls is comparable with wild type (WT) [12]. The XLXG and XLFG subunits are almost completely absent in the $x l t 2$ mutant xyloglucan, but there are only subtle phenotypic difference between $x l t 2$ and WT [5]. In $x x t 1, x x t 2$ and $x x t 5$ mutants the xyloglucan content of the wall is reduced by approximately $10 \%, 32 \%$ and 50\%, respectively, compared with WT $[8,13]$. The $x x t 1$ and $x x t 2$ mutants have no visible morphological phenotype [13] whereas $x x t 5$ forms short root hairs that have bubble-like extrusions at their tip and roots that have altered morphology [15]. Somewhat surprisingly, the xxt1xxt2 double mutant, which lacks detectable amounts of xyloglucan in its cell wall [13], only exhibits modest phenotypes including short root hairs with bulging bases [13], somewhat shorter hypocotyls and stems, and altered cellulose microfibril organization [14]. The mechanical properties of $x x t 1 x x t 2$ cell walls including wall stiffness, creep and stress relaxation also differ from WT [16]. Nevertheless, the $x x t 1 x x t 2$ mutant, which is typically a little smaller than its WT, counterpart develops normally [16]. The mur3 mutants have pleiotropic phenotypes [17]. Plants carrying the S470L (mur3-1) or the A290V (mur3-2) point mutation in MUR3 are visibly similar to WT plants $[6,18]$. However, these mutants are leaky as they synthesize xyloglucan containing discernible amounts of the F side-chain (XXFG and XLFG subunits) [19]. By contrast, mur3-3 and mur3-7, which are T-DNA knock-out lines of MUR3, are dwarfed with a cabbage-like growth phenotype, form shorter etiolated hypocotyls, and have a endomembrane aggregation phenotype [17]. The mur3-3 has also been reported to be hypersensitive to salt stress [20] and constitutively resistant to infection by Hyaloperonospora parasitica [21]. The xyloglucan from mur3-3 plants is composed of XXXG and XLXG subunits and contains no detectable amounts of the F side-chain [19].

Our previous study established that most of the mur3-3 phenotypes, including short hypocotyls, curled leaf and endomembrane aggregation is rescued by knocking out $X X T 2$ or $X X T 5$ individually or by knocking out both $X X T 1$ and $X X T 2$ [19]. The $x x t 2 m u r 3-3$ and $x x t 5$ mur3-3 mutants produce xyloglucan that is still composed of XXXG and XLXG subunits, but the XLXG subunit accounts for $60 \%$ and $72 \%$ of the xyloglucan subunits, respectively, which is almost double its abundance in mur3-3 [19]. This result suggests that the mur3-3 phenotype results from the reduced abundance of the XLXG subunit. This notion is support by the finding that overexpressing XLT2 in mur3-3 (35Spro:XLT2:mur3-3) increased the abundance of the XLXG subunit and suppressed the mur3-3 mutant phenotypes. We also found that the growth of mur3-3 was restored to near normal by abolishing their ability to synthesize xyloglucan by introducing the $x x t 1 x x t 2$ double mutation (xxt1xxt2mur3-3).

The lack of a strong phenotype in the xxt1xxt2 double mutant that forms no discernible amounts of xyloglucan has challenged the hypothesis that the strength and extensibility of the primary cell wall is controlled by a cellulose-xyloglucan 
load-bearing network [22]. The near normal appearance of this mutant has also led to questions about the role of xyloglucan in plant growth and development. Based on our previous data we proposed that galactose-deficient xyloglucan is dysfunctional and that its presence in the cell wall is more deleterious to cellular processes than the complete absence of xyloglucan.

There is increasing evidence that changes in wall structure are perceived by the cell and then relayed to the cytosol to elicit numerous responses [23]. Several proteins that may sense cell wall changes have been described including the receptor-like kinase THESEUS [24], and the pectin-binding wall-associated kinases [25]; however, the cellular signaling pathways involved are largely unknown [26]. The mur3-3 mutant provides a unique opportunity to increase our understanding of how changes in cell wall polysaccharide biosynthesis and structure affect plant growth. However, it is not known how a seemingly small change in the structure of single cell wall polysaccharides can lead to substantial growth defects. To address this question, we performed digital gene expression analysis on the etiolated hypocotyls of WT and mur3-3 plants as well as four mutant plants (xxt2mur3-3, xxt5mur3-3, xxt1xxt2mur3-3 and 35Spro:XLT2:mur3-3) in which the mur3-3 phenotypes are suppressed.

\section{Materials and methods}

\subsection{Plant materials, seeds sterilization and growth conditions}

All Arabidopsis plants used in this study were in the Columbia (Col-0) background. The homozygous T-DNA insertion mutant lines mur3-3 (At2g20370; Salk_141953), xxt2mur3-3 (XXT2, At4g02500; SALK_101308), xxt5mur3-3 (XXT5, AT1G74380; GK-411G05), xxt1xxt2mur3-3 (XXT1, At3g62720; SAIL_785_E02) and the XLT2 over-expression line 35Spro:XLT2:mur3-3 were generated as described previously [19]. Seeds were treated with aq. $10 \% \mathrm{NaClO}$ for 5 minutes and then washed three times with sterile water. Sterilized seeds were transferred to $1 / 2$ strength Murashige and Skoog agar and kept in the dark for $48 \mathrm{~h}$ at $4^{\circ} \mathrm{C}$. The seeds were then grown for 5 days at $20^{\circ} \mathrm{C}$ in the absence of light. Three replicates of sterilized seeds were prepared for each genotype. Approximately 200 hypocotyls were randomly selected from each of the three replicates and immediately frozen in liquid nitrogen prior to total RNA isolation for DGE sequencing. For hypocotyl length measurement, images of at least ten seedlings of each genotype were obtained using a Canon 5D Mark III digital camera. The length measurement of hypocotyls was performed using Image $\mathbf{J}$ software [27].

\subsection{RNA extraction, preparation of digital expression libraries and Illumina sequencing}

Total RNA was extracted with QIAGEN RNeasy ${ }^{\circledR}$ Plant Mini Kit (QIAGEN, Cat. NO. 74903). DNase was used to digest genomic DNA. RNA purity was assessed using a NanoPhotometer ${ }^{\circledR}$ spectrophotometer (IMPLEN, CA, USA). The amounts of RNA were measured using a Qubit ${ }^{\circledR}$ RNA Assay Kit (Life Technologies, CA, USA) and RNA integrity was assessed using a RNA Nano 6000 Assay Kit of the Bioanalyzer 2100 system (Agilent Technologies, CA, USA). A total of $3 \mu \mathrm{g}$ purified RNA from each plant line was used to generate the Illumina sequencing libraries using a NEBNext ${ }^{\circledR}$ Ultra $^{\mathrm{TM}}$ RNA Library Prep Kit for Illumina ${ }^{\circledR}$ (NEB, USA) 
following the manufacturer's recommendations. The libraries were sequenced using a Illumina Hiseq 2000/2500 platform by Beijing Novogene Bioinformatics Technology Co. Ltd (Beijing, China).

\subsection{Analysis of digital gene expression reads and identification of expressed genes}

The raw data was filtered to eliminate reads containing adapter, ploy- $\mathrm{N}$ and low quality reads. The Q20, Q30 and GC content of the cleaned data were then calculated to determine the quality of the reads. The single-end clean reads were then mapped to the A. thaliana reference genome using TopHat v2.0.9 [28]. Only reads with a perfect match or at most two bases of mismatch were permitted in the alignment. HTSeq v0.6.1 was used to calculate the number of reads mapped to each gene (read counts data) [29]. Reads Per Kilobases per Million mapped reads (RPKM), which considers the effect of sequencing depth and gene length for the read counts at the same time and is currently the most commonly used method for estimating gene expression levels in DGE analysis [30], was used to estimate the expression level of each gene. In this study, the expressed gene was defined by RPKM value greater than 1 .

\subsection{Differential expression analysis}

Prior to differential gene expression analysis for each sequenced library, the read counts data were adjusted by the TMM method [31]. Differential expression analysis of two libraries was performed using the DEGSeq R package (1.12.0) [32]. The P-values, which indicate significant differences of transcript accumulation was adjusted using the Benjamini and Hochberg method [33]. A combination of the corrected P-value $<0.005$ and the absolute value of $\log _{2}$ (fold change) $\geq 1$ were used as the thresholds to assess the significance of differences in gene expression.

\subsection{GO, KEGG and MapMan enrichment analysis and gene classification analysis}

GO functional categories were assigned to the differentially expressed genes using the web-based agriGO tools [34]. GO enrichment analysis of DEGs was implemented using singular enrichment analysis (PAGE) by comparing a query list of differentially expressed genes to a background gene set (FDR $<0.05$ was as criteria). KOBAS software [35] was used for pathway enrichment analyses by testing the statistical enrichment of DEGs in KEGG pathways. MapMan tool [36] was used for overview of metabolic pathways, regulatory processes and biotic stress processes.

\subsection{Annotation of TFs and cell wall-related genes}

TF family members were annotated based on a published data [37]. Cell wall-related genes were annotated based on the Purdue Cell-Wall-Genomics Database (http://cellwall.genomics.purdue.edu/).

\subsection{Promoter conserved motifs and WGCNA analysis}

72 down-regulated and 30 up-regulated DEGs in mur3-3 compared with WT and the four suppressor lines were used for promoter conserved motifs analysis. $2 \mathrm{~kb}$ length promoters of the 102 overlapped DEGs were analyzed using the web based tool (http://www.bioinformatics2.wsu.edu/cgi-bin/Athena/cgi/home.pl). The occurrence of detected conserved motifs was further screened using the P-value result 
(P-value result $<0.05$ was as criteria). The weighted gene co-expression network analysis (WGCNA) was performed in R using the WGCNA package, as previously described [38]. Differentially expressed genes including cell wall-related genes, hormone-related genes, TFs, genes encoding the kinase protein, aquaporin and chlorophyll a-b binding protein were used to construct an unsupervised co-expression based similarity matrix using Pearson's correlation coefficient. The $\mathrm{R}$ package WGCNA version 1.35 was then used to create the networks [38], which were visualized with Cytoscape 3.2 [39].

\subsection{Plant transformation and complementation test}

A total of 11 DEGs including cell wall-related genes, TFs, hormone-related genes, kinase genes and an aquaporin gene, which were down-regulated in mur3-3 compared with WT and the suppressor lines were overexpressed in mur3-3. The full-length CDS sequences of each gene were cloned into the XbaI and KpnI sites downstream of the cauliflower mosaic virus $35 \mathrm{~S}$ promoter in the pCam35tlegfps $2 \# 4$ vector [19]. The constructs were introduced into Agrobacterium strain GV3101, and the bacteria were used to transform mur3-3 mutant plants by a floral dip transformation method [40]. Transgenic plants were selected by growth on $1 / 2$ strength MS medium containing hygromycin $(15 \mathrm{mg} / \mathrm{L})$. The presence of the transgene was confirmed by PCR using the gene-specific primer paired with vector-specific primer. The hygromycin resistant $\mathrm{T}_{1}$ transgenic plants and mur3-3 seedlings were then grown in controlled-environmental growth chambers at $19^{\circ} \mathrm{C}$ with a 16 -h-light and 8 -h-dark cycle for phenotype observation. Seeds treatment and hypocotyl measurement of $\mathrm{T}_{2}$ transgenic plants were the same as described in 2.1 above.

\subsection{Quantitative RT-PCR}

RNA for qRT-PCR analysis was extracted from the hypocotyls of six plants as described above. First-strand cDNA was synthesized from $1 \mu \mathrm{g}$ of DNA-free RNA using a TransScript ${ }^{\circledR}$ One-Step gDNA Removal and cDNA Synthesis SuperMix Kit (TransGen, AT311-02) according to the manufacturer's instructions. The qRT-PCR mixture $(20 \mu \mathrm{L}$ total volume) contained $10 \mu \mathrm{L}$ of SYBR Green super mix (Roche Diagnostics $\mathrm{GmbH}$, Mannheim, Germany), $3 \mu \mathrm{L}$ of each primer $(2 \mu \mathrm{M}), 1 \mu \mathrm{L}$ of cDNA, and $3 \mu \mathrm{L}$ of RNase-free water. The reactions were performed on an Applied Biosystems 7500 Real-Time PCR system according to the manufacturer's instructions. All reactions were performed in three biological replicates with three technical replicates each. Expression was calculated as $2^{-\Delta \Delta C t}$ [41] and normalized to that of the TUBLIN gene. The primer sets were designed using Primer-BLAST (http://www.ncbi.nlm.nih.gov/tools/primer-blast/) to avoid non-specific amplification. Primers used in this study were shown in Supplementary File 1.

\section{Results}

\subsection{DGE library sequencing and mapping sequences to the reference genome}

The mur3-3 mutant has a significantly shorter hypocotyl length than WT. Hypocotyl length is restored to near normal in each of the four suppressor lines (Fig. 1). To investigate the gene expression patterns in these six plant lines, RNA was extracted from the hypocotyls and sequenced using the Illumina Hiseq 2000/2500 
platform. After the removal of adaptor sequences, duplicate sequences, ambiguous reads and low-quality reads we obtained broadly similar numbers of cleaned reads from WT (14789989), mur3-3 (10793421), xxt2mur3-3 (16686602), xxt5mur3-3 (11551898), xxt1xxt2mur3-3 (10225651), and 35Spro:XLT2:mur3-3 (12207798) (Table 1). The average percentage ratio of clean reads was $98.25 \%$ among the six libraries. The average Q20 percentage of the six libraries was $98.98 \%$ and the average GC was $45.52 \%$ (Table 1). Saturation and mean coverage analysis results showed that the DGE-seq data reads were distributed uniformly over the transcript and that their quality was suitable for quantitative expression analysis (Fig. S1). The cleaned reads from WT (14350871), mur3-3 (10446763), xxt2mur3-3 (16213637), xxt5mur3-3 (11172751), xxt1xxt2mur3-3 (9887405), and 35Spro:XLT2:mur3-3 (11790956) were mapped to the $A$. thaliana genome with mapping ratios between $96.6 \%$ and $97.2 \%$ (Table 1). Similar numbers of genes were identified for WT (18123), mur3-3 (18384), xxt2mur3-3 (18242), xxt5mur3-3 (18100), xxt1xxt2mur3-3 (18101), and 35Spro:XLT2:mur3-3 (18468). A total of 19,549 genes were expressed in all six plant lines of which 16,938 were overlapped (Supplementary File 2). Based on the RPKM data, only a small proportion of the genes were highly expressed. For example, only 61 of the 18123 WT genes had an expression value of more than $3\left(\log _{10}(\mathrm{RPKM}+1)\right)$ (Fig. 2). All six libraries had similar RPKM distributions, which suggest that their transcript profiles are comparable (Fig. 2).

\subsection{Overview of differentially expressed genes}

To identify key genes involved in hypocotyl cell elongation in the xyloglucan-related mutants, we selected genes that are differentially expressed by using a corrected P-value $<0.005$ and an absolute value of $\log _{2}$ (fold change) $\geq 1$ as the thresholds. Of the 1423 DEGs that differed between WT and mur3-3, 676 were up-regulated and 747 were down-regulated. 19 DEGs were uniquely expressed in WT and 50 in mur3-3 (Fig. 3, Supplementary File 3). In the comparison between xxt2mur3-3 and mur3-3, 283 DEGs were up-regulated and 392 were down-regulated. Only two genes were uniquely expressed in mur3-3 (Fig. 3, Supplementary File 3). Similar numbers of DEGs were up- (613) or down-regulated (659) in xxt5mur3-3 vs mur3-3, with 15 uniquely expressed in xxt5mur3-3 and 17 in mur3-3 (Fig. 3, Supplementary File 3). In the xxt1xxt2mur3-3 vs mur3-3 comparison 574 DEGs were up-regulated and 623 down-regulated, with 10 uniquely expressed in xxt1xxt2mur3-3 and 38 in mur3-3 (Fig. 3, Supplementary File 3). 79 DEGs were up-regulated and 42 down-regulated in the 35Spro:XLT2:mur3-3 vs mur3-3 comparison, but no genes were uniquely expressed in either plant (Fig. 3, Supplementary File 3). Together the results of our analyses identified 887 up-regulated and 914 down-regulated genes whose expression was significantly different in the five comparisons. The up- and down-regulated genes can be clearly distinguished in the hierarchical clustering map which classified 1801 differential expression genes based on the RPKM value (Fig. 4). It is notable that xxt1xxt2mur3-3 most closely resembles WT, as these two lines differed by only 99 DEGs (Supplementary File 3). Thus, the elimination of xyloglucan may have less effect on gene expression than the formation of a dysfunctional xyloglucan.

\section{3. $G O$ and KEGG annotation of differentially expressed genes}


We next used GO and KEGG assignments to annotate the differentially expressed genes to reveal their potential functions. Numerous significantly enriched GO terms of biological process, molecular function and cellular component were categorized in comparisons of mur3-3 with WT (151), xxt2mur3-3 (159), xxt5mur3-3 (197), and with xxt1xxt2mur3-3 (131) (Supplementary File 4, Fig. 5). No significantly enriched GO terms were categorized in the comparison of mur3-3 with 35Spro:XLT2:mur3-3. In the cellular component category, "plastid", "plastid part", "chloroplast", and "organelle part" were significantly enriched (p-value $<0.05)$ in comparisons of mur3-3 with WT, xxt2mur3-3, xxt5mur3-3, and with xxt1xxt2mur3-3 (Supplementary File 4, Fig. 5). However, in the molecular function category, the top four enriched GO terms showed difference among these four comparisons. "Structural constituent of ribosome", "structural molecule activity", "oxygen binding", and "transferase activity, transferring glycosyl groups" were dominantly enriched in WT vs mur3-3. However, the four most dominant enriched GO terms in xxt1xxt2mur3-3 vs mur3-3 were "hydrolase activity, acting on acid anhydrides, in phosphorus-containing anhydrides", "hydrolase activity, acting on acid anhydrides", "nucleoside-triphosphatase activity", and "pyrophosphatase activity". GO terms related to "tetrapyrrole binding", "passive transmembrane transporter activity", "substrate-specific channel activity" and "channel activity" were enriched in $x x t 2$ mur3-3 vs mur3-3, which is a little difference with the most dominantly enriched four GO terms in xxt5mur3-3 vs mur3-3 "transcription factor activity", "DNA binding", "transcription regulator activity", and "structural constituent of ribosome" (Supplementary File 4, Fig. 5). In the biological process category, "immune system process", "immune response", "innate immune response", and "photosynthesis" were dominantly enriched both in WT vs mur3-3 and $x x t 1 x x t 2 m u r 3-3$ vs mur3-3. However, the top four enriched GO terms in $x x+5$ mur3-3 vs mur3-3 were "photosynthesis", "immune system process", "immune response", and "cellular metabolic process". "Photosynthesis", "cellular metabolic process", "metabolic process", and "cellular process" were dominantly enriched in xxt2mur3-3 vs mur3-3, which showed a little difference with xxt5mur3-3 vs mur3-3 (Supplementary File 4, Fig. 5). In addition, we observed that the vast majority DEGs which enriched into immune response related GO terms were down-regulated in WT, xxt1xxt2mur3-3 and $x x+5$ mur3-3 compared with mur3-3 (Supplementary File 5).

DEGs from comparisons of mur3-3 with WT (897), xxt2mur3-3 (517), xxt5mur3-3 (827), xxt1xxt2mur3-3 (80) and 35Spro:XLT2:mur3-3 (84) were mapped to 97, 79, 92, 87 and 31 KEGG pathways, respectively (Supplementary File 6). The top twenty significantly enriched KEGG pathways of each comparison are shown in Fig. S2. Metabolic pathways (average 22\% of total mapped DEGs of every comparison) and the biosynthesis of secondary metabolites (average 12\% of total mapped DEGs of every comparison) were most represented by the DEGs. Notably, we also observed that pathways related to "brassinosteroid biosynthesis" and "plant hormone signal transduction" were enriched in all five comparisons. These annotations provide a valuable resource for investigating specific processes, functions and pathways related with cell elongation.

\subsection{Differential expression genes involved in cell wall synthesis and modification}

A total of 127 DEGs related to cell wall synthesis or modification showed different transcript levels in a comparison between mur3-3, WT and the four suppressor lines. This included genes encoding xyloglucan 
endotransglucosylase/hydrolases (XTHs), expansins, pectin methyl esterases (PMEs), arabinogalactan-proteins (AGPs), peroxidases, ß-galactosidase, cellulose synthases and glucan endo-1,3-beta-glucosidase (Supplementary File 7). Comparisons of mur3-3 with WT, xxt2mur3-3, xxt5mur3-3, xxt1xxt2mur3-3 or 35Spro:XLT2:mur3-3 identified 18 DEGs that included three XTHs (XTH4, XTH17 and XTH3O), four AGPS (AGP5, AGP22, AGP24 and FLA13), three PMEs (PME3, PME16 and PME35) as well as EXPA8, PER53, BCB1 and COMT1. All of these genes were up-regulated in WT and the four suppressor lines compared with mur3-3 except AGP5, PER53 and $B C B 1$, which were down-regulated in WT and four suppressor lines compared with mur3-3. An additional 28 DEGs were detected in comparisons of mur3-3 with WT, $x x t 2 m u r 3-3, x x t 5 m u r 3-3$, or $x x t 1 x x t 2 m u r 3-3$ and included six AGPs (AGP1, AGP4, AGP7, AGP13, AGP14 and AGP21), two EXPAs (EXPA6 and EXPA11), two PMEs (PME34 and PME41), two PERs (PER64 and PER72), two BGALs (BGAL4 and $B G A L 8)$ as well as CESA2, CSLA9, XTH33, E13A, ENL2, GLPQ3, ATRABE1B. These DEGs were all up-regulated in WT and four suppressor lines compared with mur3-3 except AGP1, ATRABE1B, EXPA6, GLPQ3, PME41 and ENL2, which were down-regulated in WT and four suppressor lines compared with mur3-3. The remaining 81 cell wall-related DEGs were detected in other comparison pairs. 48 were up-regulated and 33 down-regulated in WT and four suppressor lines compared with mur3-3.

\subsection{Differential expression genes involved in transcriptional factors}

Transcription factors (TFs) have a key role in controlling gene expression in plants. We identified 130 differentially expressed TFs that belong to 24 different families including AP2-EREBP, ARF/Aux-IAA, bZIP, MYB, WRKY, bHLH, C2H2, C2C2 (Zn), NAC and Homeobox (Supplementary File 8). Three TFs (IAA3, MYB88 and a member of the $\mathrm{C} 2 \mathrm{H} 2$ gene family) were all up-regulated in WT and the four suppressor lines compared with mur3-3. 21 TFs were differentially expressed in comparisons between mur3-3 and WT, xxt2mur3-3, xxt5mur3-3 or xxt1xxt2mur3-3. Two ARF/Aux-IAA TFs (IAA6 and IAA17), four bHLH TFs (BHO36, BH078, BH151 and AT2G40820), two Homeobox TFs (HAT1 and HAT2), MYB86 and NAClOO were up-regulated in WT, xxt2mur3-3, xxt5mur3-3 or xxt1xxt2mur3-3 compared with mur3-3. By contrast, three $C 2 C 2(\mathrm{Zn}) \mathrm{TFs}(\mathrm{COL6}, \mathrm{COL} 8$ and COL16), two C2H2 TFs (ZAT8 and ZAT12) were down-regulated in WT, xxt2mur3-3, xxt5mur3-3 or xxt1xxt2mur3-3 compared with mur3-3. The remaining 106 TFs detected in comparisons between mur3-3 and the other lines, included 83 that were up-regulated and 23 that were down-regulated in WT or some suppressor lines compared with mur3-3.

\subsection{Detection of overlapped DEGs}

The "cabbage-like" phenotype of mur3-3 is suppressed by preventing xyloglucan synthesis (xxt1xxt2mur3-3) or by increasing the abundance of the XLXG subunit (xxt2mur3-3, xxt5mur3-3 and 35Spro:XLT2:mur3-3). To explore the underlying mechanism of suppression, we used Venn map statistical analysis to identify the overlapped DEGs (Fig. 6). A total of 102 overlapped DEGs were identified in comparisons of mur3-3 with WT, xxt2mur3-3, xxt5mur3-3, xxt1xxt2mur3-3 or 35Spro:XLT2:mur3-3. 72 were up-regulated and 30 were down-regulated in WT and four suppressor lines compared with mur3-3 (Supplementary File 9, Fig. 6A). 18 of 
these genes, including XTH4, XTH17, XTH30, PME3, PME61, EXPA8, AGP5, $A G P 22$ and $A G P 24$ are involved in cell wall biosynthesis or modification. Almost all of these cell wall-related DEGs were up-regulated (Table 2). 95\% of the DEGs (115/121) detected in 35Spro:XLT2:mur3-3 vs mur3-3 were also detected in WT vs mur3-3 (Supplementary File 3).

A total of 550 overlapped DEGs were identified in comparisons of mur3-3 with WT, $x x t 2 m u r 3-3, x x t 5$ mur3-3 or $x x t 1 x x+2 m u r 3-3.227$ were up-regulated and 323 were down-regulated in comparisons with mur3-3 (Supplementary File 9, Fig. 6B). 24 TFs and 46 cell wall-related DEGs, which include the 18 cell wall-related DEGs as described above, were identified. In addition, 6 auxin and brassinosteroids (BR) related genes, 9 protein kinase genes, 9 aquaporin genes and 18 chlorophyll a-b binding protein genes were identified. Notably, most of cell wall-related DEGs, TFs, hormone-related genes, protein kinase and aquaporin genes were up-regulated in WT and suppressor lines compared with mur3-3, whereas all of the chlorophyll a-b binding protein genes were down-regulated in WT and suppressor lines compared with mur3-3. Visualization of the 550 DEGs using MapMan revealed a clustering of these genes into metabolic pathways (Fig. 7A), regulatory processes (Fig. 7B), and biotic stress processes (Fig. 7C). Most cell wall-related genes, protein modification genes, hormone-related genes and PR-protein genes were up-regulated relative to mur3-3, while all the light reaction related genes were down-regulated relative to mur3-3.

\subsection{Conserved promoter motifs analysis}

Conserved motifs in gene promoters typically indicate related functions of a cluster of genes. To explore the potential variance of gene function between the upand down-regulated DEGs, we analyzed the conserved promoter motifs of the 102 overlapped DEGs detected in the comparison pairs of WT, xxt2mur3-3, xxt5mur3-3, xxt1xxt2mur3-3 and 35Spro:XLT2:mur3-3 vs mur3-3. A total of 27 and 12 conserved promoter motifs were significantly enriched in up- and down-regulated DEGs (P-value<0.05), respectively (Supplementary File 10). A TATA-box, CARGCW8GAT, T-box promoter, Ibox promoter, ABRE-like binding site and Z-box promoter motifs were detected in most of the up- and down-regulated DEGs. 21 and 6 conserved promoter motifs were specifically detected in up- and down-regulated DEGs, respectively. For example, MYB1AT, the MYB4 binding site and W-box promoter motifs, GAREAT, MYCATERD1 and AtMYC2 BS in RD22 conserved promoter motifs were specifically enriched in up-regulated DEGs. SV40 core promoter motif, ACGTABREMOTIFA2OSEM and GADOWNAT were enriched in down-regulated DEGs.

\subsection{Complementation test}

We selected 11 DEGs down-regulated in mur3-3 relative to WT and the four suppressor lines to investigate their role in cell elongation. The DEGs encode cell wall-related proteins, TFs, hormone-related proteins, protein kinases and aquaporins (Supplementary File 11). Each DEG was overexpressed in mur3-3 to determine if they rescued the mur3-3 phenotype. At least 20 transgenic lines were generated for each gene. The aerial portions of the transgenic lines and mur3-3 were indistinguishable (data not shown) suggesting that the leaf growth phenotype cannot 
be rescued by expressing any of these genes individually. Two transgenic lines (35Spro:IAA6:mur3-3 and 35Spro:IAA17:mur3-3) had shorter hypocotyls than mur3-3 (Fig. 8). By contrast, the mur3-3 short hypocotyl phenotype was partially rescued by overexpressing four cell wall-related genes (XTH4, XTH3O, PME3 and EXPA11), a TF (MYB88), a hormone-related gene (ROT3), two protein kinase genes (AT5G37790 and WAG2) and a aquaporin gene (TIP2;3) (Fig. 8). Thus, differences may exist in cell elongation mechanism in adult plants and in hypocotyls.

\subsection{Co-expression networks underlying the hypocotyl elongation}

The 46 cell wall-related genes, 24 TFs, 6 hormone-related genes, 9 kinase genes, 9 aquaporin genes and 18 chlorophyll a-b binding protein genes showed different expression levels in mur3-3 compared with WT, xxt2mur3-3, xxt5mur3-3 or xxt1xxt2mur3-3 (Supplementary File 9). Complementation test showed that elevating some of these genes that were down-regulated in mur3-3 partially rescued the hypocotyl phenotype (Fig. 8). We constructed a global co-expression network of these genes by using WGCNA to explore the potential correlations of these DEGs with the regulation of cell elongation. These results showed that most of the cell wall-related genes including XTH33, EXPA6, PRX53, BGL2 and ENODL2 co-expressed with TFs, hormone-related genes and genes encoding protein kinases, aquaporins, and chlorophyll a-b binding proteins (Supplementary File 12, Fig. 9).

In addition, among these co-expression DEGs, the TFs MYB55 (AT4G01680), BBX17 (AT1G49130), RING1 (AT5G10380) and AT3G46080, hormone-related genes CYP710A2 (AT2G34490) and ROT3 (AT4G36380) had expression patterns (WT>xxt5mur3-3>xxt2mur3-3>mur3-3) similar to genes encoding aquaporin proteins PIP1;1 (AT3G61430), PIP2;2 (AT2G37170), PIP2;4 (AT5G60660) and TIP2;2 (AT4G17340) and kinase proteins AT3G15356 and GPK1 (AT3G17420) (Supplementary File 9, Supplementary File 12). The expression level trend of these DEGs is correlated with the abundance of the XLXG subunit in the plants, indicating that expressions of the DEGs are correlated with the presence of the dysfunctional xyloglucan. This result further supports our previous conclusion that the mur3-3 phenotype results from the reduced amounts of the XLXG subunit.

\subsection{Quantitative real-time PCR analysis}

To validate the differential DEG data obtained by Illumina Hiseq 2000/2500 platform sequencing we used qRT-PCR analyses of eleven cell-wall related DEGs, one TF and three protein kinase DEGs selected randomly from the 550 overlapped DEGs detected from comparisons of mur3-3 with the other plants (Fig. 6B). The qRT-PCR expression patterns of these fifteen DEGs exhibited similar expression patterns with DGE-seq (Fig. 10A). Linear regression analysis showed a significant correlation $\left(\mathrm{R}^{2}=0.9323\right)$, which indicates good reproducibility between transcript abundance generated by DGE-seq and the expression profiles obtained from the qRT-PCR data (Fig. 10B).

\section{Discussion}

To our knowledge, our study is the first to provide a large-scale assessment of the transcriptomes of plants carrying mutations in GTs known to be involved in the 
synthesis of xyloglucan. This study provides a foundation for understanding the underlying mechanism of how changes in xyloglucan structure affect cell elongation.

Cell wall synthesis and remodeling are key steps in the assembly of a functional cell wall and thus have important roles in regulating cell elongation and division. Several genes involved in these two processes including those encoding glycoside hydrolases, expansins, carbohydrate lyases, peroxidases, laccases, AGPs, fasciclin AGPs (FLAs), PMEs, PMEIs, GHs, XTHs, beta-galactosidases, cellulose and callose synthase genes (Supplementary File 7) were identified in our study and found to be differentially expressed.

Xyloglucan and pectin have been proposed to have key roles in determining the mechanical properties of the primary cell walls [42] and thus are important for normal cell elongation. Thus, it is not unexpected that genes encoding PMEs (5) and XTHs (3) were present as overlapped DEGs in pairwise comparisons of WT vs mur3-3, $x x t 2 m u r 3-3$ vs mur3-3, xxt5mur3-3 vs mur3-3 and $x x t 1 x x t 2 m u r 3-3$ vs mur3-3. Many of these genes were also identified to have important roles during cell elongation and hypocotyl growth in transcriptomics or proteomics studies of elongating Arabidopsis hypocotyls [43-45]. Our complementation test supports this notion since overexpression of XTH4, XTH3O, PME3 and EXPA11 partially rescued the length of mur3-3 hypocotyls (Fig. 8).

It is intriguing that the $x x t 1 x x t 2 m u r 3-3$ mutant, which lacks xyloglucan in its cell walls has gene expression profiles that most closely resemble WT (Supplementary File 2). This adds support to our previous proposal that the absence of xyloglucan has a less deleterious effect on plant growth than the presence of xyloglucan with a dysfunctional structure [19]. In comparison to mur3-3, the expression level of more than $80 \%$ of DEGs in WT vs mur3-3 reverted to WT levels in xxt2mur3-3, xxt5mur3-3, xxt1xxt2mur3-3 and even 35Spro:XLT2:mur3-3 (Supplementary File 13), which suggests that in these suppressor lines the mur3-3 phenotypes are suppressed through similar pathways and that the galactose-deficient xyloglucan or galactose-deficient xyloglucan oligosaccharides signal the cellular changes and thus affect cell elongation.

\subsection{Auxin and BR may contribute to mur3-3 hypocotyl elongation defect}

Auxin regulates many aspects of plant growth and development [46]. This hormone controls transcription by rapidly modulating the levels of short-lived Aux/IAA proteins [47]. In the $\mathrm{SCF}^{\mathrm{TIR} 1 / \mathrm{AFB}}$-auxin-Aux/IAA auxin signaling transduction model, auxin triggers Aux/IAA degradation and de-repression of ARF activity, enabling promoter activation for gene expression [47]. In our study, we found that IAA3, IAA6 and IAAI7 were significantly down-regulated in mur3-3 compared with WT (Supplementary File 8) together with the down-regulation of $X T H s, P M E s$ and AGPs (Table2, Supplementary File 7). It is notable that the expression levels of the three Aux/IAAs genes in the mur3-3 suppressor lines revert to WT levels (Supplementary File 2). Several hormones, including auxin and BR, have been proposed to mediate their effects on cell elongation via alteration of wall architecture [48]. Thus, it is possible that the changes in cell wall-related DEGs are regulated by changes in IAA. 
Several studies have reported that seedlings of the gain-of-function mutants shy2-2/iaa3, shyl/iaa6 and axr3/iaa17 have short hypocotyls and curled leaves and that genes encoding XTHs, PMEs, and AGPs are repressed [49-52]. The XTH17, $A G P 22$, and AGP4 genes that are repressed in axr3/iaal7 were also repressed in mur3-3 compared with WT. Additional experiments are required to verify if gain-of-function of IAAs and knock down of IAAs inhibit plant growth through similar pathways. Notably, our overexpression experiments showed that IAA6 and IAA7 do not rescue, but enhance the mur3-3 short hypocotyl phenotype further implicating the complex regulatory mechanism of auxin during hypocotyl elongation.

Brassinosteroids are a class of hormones that regulate numerous aspects of plant growth and development [53]. These hormones are perceived by BRI1, which then activates the TFs BZRl/2 through inhibition of BIN2, a GSK3-like kinase. BZRl/2, also regulates numerous other genes including many involved in cell wall-biosynthesis and remodeling $[54,55]$. Previous studies reported that many BR biosynthetic mutants including $\operatorname{det} 2$, dim, dwarfl, cbbl, brdl, lka and $l k b$ have short hypocotyls, petioles, and stems, and small leaves [56-59] suggesting that the dwarfism in BR biosynthetic and BR-insensitive mutants results from reduced cell elongation. Consistent with previous studies, we found that three genes involved in sterol biosynthetic (ROT3, CYP710A2 and CYP710A1) were down-regulated in mur3-3 compared with WT, xxt2mur3-3, xxt5mur3-3, xxt1xxt2mur3-3 and 35Spro:XLT2:mur3-3. Since most BR biosynthetic genes are believed to be under negative feedback control by BR signaling [48], it is possible that the elongation defects in mur3-3 result in part from elevated BR signaling. A previous study has reported that inhibition of PME activity leads to a reduction of pectate in the cell wall, which may trigger BR signaling. Elevated BR signaling is the main cause of the abnormal phenotype when PME activity is disturbed [48]. Consistent with such studies, we found that PME3, PME16, PME34 and PME35 were all down-regulated in mur3-3 relative to WT (Table 2). Thus, in mur3-3, the reduced expression of PMEs may result in elevated BR signaling which then leads to the "cabbage-like" phenotype. Evidence to support this notion was obtained by complementation tests, which showed that the short hypocotyl phenotype of mur3-3 is partially rescued in the 35Spro:PME3:mur3-3 and 35Spro:ROT3:mur3-3 transgenic lines (Fig. 8).

4.2. TFs and receptor kinase proteins that possibly responsible for hypocotyl elongation defect in mur3-3

Plasma membrane-localized receptor-like kinases (RLKs) have been suggested to be the primary candidates for sensing changes in cell wall integrity [23]. Wall associated kinases (WAKs) that have a highly conserved serine/threonine protein kinase domain on their cytoplasmic domain and the Catharanthus roseus RLK1 (CrRLK1) like protein family are two of the best-studied potential cell wall integrity receptors $[60,61]$. In our study, we identified 9 kinases in the 550 overlapped genes, including the CrRLK1-like family member HERCULES1 (HERK1) and 8 serine/threonine protein kinases. Further experiments are required to verify whether these two RLKs are involved in sensing changes in cell wall mechanics that result from altered xyloglucan structure. Indeed, our data showing that overexpression WAG2 or At5g37790 partially rescued the mur3-3 short hypocotyl phenotype suggests 
that kinases do have a role in xyloglucan signal sensing and transduction.

Identifying TFs responsible for the mur3-3 short hypocotyl phenotype is required to elucidate the underlying mechanism that control cell elongation. In our study, several TFs gene families including bHLH, MYB, homeobox, BZR, NAC, Sigma70-like and the trihelix family were identified (Supplementary File 8). Overexpression of MYB88 partially rescued the mur3-3 hypocotyl phenotype, suggesting that one or more of these TFs are potential candidates for the cell elongation defect observed in mur3-3 hypocotyls.

\subsection{Aquaporins may facilitate cell elongation}

Plant cell growth requires the irreversible increase in the surface area of the cell wall. This process requires an internal turgor pressure, which is generated by water uptake into the cell [62-64]. To maintain turgor during cell wall expansion, water movement through cell membranes is enabled by water channels referred to as aquaporins [65]. Aquaporins are integral membrane proteins that form pores which facilitate water transport at a rate higher than diffusion. The increased accumulation of aquaporins likely allows water to enter a cell at an accelerated rate, leading to a higher turgor pressure that drives cell elongation. Consistent with this hypothesis, a previous report showing that transgenic tobacco overexpressing an Arabidopsis plasma membrane aquaporin gene $(P I P I b)$, are taller than WT as a consequence of the increased length and number of internodes [66]. We found that seven PIPs and two TIPs aquaporin genes were up-regulated in WT and the four suppressor lines compared with mur3-3 (Supplementary File 9), suggesting that these proteins may help maintain a higher turgor pressure to facilitate the normal cell elongation. This notion was supported by complementation tests showing that overexpressing TIP 2;3 in mur3-3 promoted cell elongation. On the other hand, it has been reported that in Arabidopsis roots auxin suppresses the transcription of several PIP and TIP genes including PIP1;1, PIP 1;5, PIP2;1, PIP2;2, PIP2;4, PIP2;7, TIP2;2 and TIP2;3 [67]. These 8 genes also appeared in the 550 overlapped DEGs and were down-regulated in mur3-3 compared with WT and the suppressor lines (Supplementary File 9). Thus we suggest that the lower expression of these aquaporin genes in mur3-3 is due to an elevated auxin signal since IAA3, IAA6, IAAI7 were significantly down-regulated in mur3-3.

\subsection{The elevated immune response in mur3-3}

Plant cell walls serve as physical barriers against pathogen entry, however, phytopathogenic fungi, bacteria, and nematodes secrete enzymes that can break the integrity of the plant cell wall [68]. Pectic enzymes, including polygalacturonases (PGs), pectate lyases (PELs) and PMEs are typically the first cell wall degrading enzymes secreted by fungal and bacterial pathogens during the initial stages of infection [69]. Xyloglucan, the predominant hemicellulosic polysaccharide in the primary walls of dicots and non-graminaceous monocots, is fragmented by microbial xyloglucan-specific endoglucanases (XEGs) [68]. Plant cells have membrane-localized receptors that perceive the molecules generated by the cell wall-degrading enzymes in damaged tissues [70] and activate plant immune responses $[71,72]$. In our study, we found that several GO terms, including "immune system process", "immune response" and "innate immune response" were enriched in mur3-3 
compared with WT or xxt1xxt2mur3-3. The latter two terms were also enriched in mur3-3 compared with $x x t 5$ mur3-3 (Supplementary File 4, Fig. 5). Most of the DEGs enriched in these GO terms were up-regulated in mur3-3 compared with WT or suppressor lines (Supplementary File 5) indicating that the dysfunctional xyloglucan in mur3-3 activates severe immune response, although further studies are needed to clarify the underlying mechanism.

The esterification status of pectin can affect cell wall degradability by microbial enzymes. Genetic and molecular evidence has indicated that pectin methyl-esterification has a critical role in a plant's defence against pathogens [73]. Studies in Arabidopsis and wheat have shown that lower levels of PME activity is correlated with an increase of pectin esterification and a concomitant decrease in susceptibility to the pathogen $[74,75]$. In our data, several genes encoding PMEs were down-regulated in mur3-3 compared with WT and the suppressor lines (Supplementary File 7). The constitutive resistance of mur3-3 petioles to infection by $H$. parasitica [21] may result from elevated expression of these immune response genes and the decreased expression of PME genes.

\section{Conclusions}

Our DGE-seq data provides insights into the molecular basis of how modifying a cell wall polysaccharide may lead to reduced cell elongation. Structural changes to cell wall xyloglucan must be perceived and then relayed to the cytosol where they cause changes in the expression of numerous genes related to cell wall metabolism, TFs, hormones, receptor kinases and aquaporins. Our study illustrates how combining knowledge of mutant cell wall structure with gene expression data and bioinformatics provides new insight into the molecular mechanism underlying plant cell elongation.

\section{Acknowledgements}

This work was supported by the National Natural Science Foundation of China (31470291, 31670302), the National Key Technology R\&D Program (2015BAD15B03-05), the Elite Youth Program of CAAS (to YK), the Taishan Scholar Program of Shandong (to GZ) and The Division of Chemical Sciences, Geosciences, and Biosciences, Office of Basic Energy Sciences of the U.S. Department of Energy through grant DE-FG02-12ER16324 (to MAO).

\section{Authors' contributions}

This study was conceived by ZX, DS, TN and YK. The preparation of plant material was conducted by DS, TN and MW. Complementation test were finished by MW. ZX, MW, GZ and YK contributed to the data analysis, the bioinformatics analysis and manuscript preparation. MAO and $\mathrm{MGH}$ revised the manuscript. All authors have read and approved the final manuscript. 


\section{References}

[1] M.A. O'Neill,W.S. York, The composition and structure of plant primary cell walls, The plant cell wall. Blackwell Publishing/CRC Press, Ithaca, New York (2003) 1-54.

[2] M.J. Pena, A.G. Darvill, S. Eberhard, W.S. York,M.A. O'Neill, Moss and liverwort xyloglucans contain galacturonic acid and are structurally distinct from the xyloglucans synthesized by hornworts and vascular plants, Glycobiology. 18 (2008) 891-904.

[3] R.M. Perrin, Z. Jia, T.A. Wagner, M.A. O'Neill, R. Sarria, W.S. York, N.V. Raikhel,K. Keegstra, Analysis of xyloglucan fucosylation in Arabidopsis, Plant Physiol. 132 (2003) 768-778.

[4] M. Hoffman, Z. Jia, M.J. Peña, M. Cash, A. Harper, A.R. Blackburn II, A. Darvill,W.S. York, Structural analysis of xyloglucans in the primary cell walls of plants in the subclass Asteridae, Carbohydrate Research. 340 (2005) 1826-1840.

[5] J.K. Jensen, A. Schultink, K. Keegstra, C.G. Wilkerson,M. Pauly, RNA-Seq analysis of developing nasturtium seeds (Tropaeolum majus): identification and characterization of an additional galactosyltransferase involved in xyloglucan biosynthesis, Mol Plant. 5 (2012) 984-992.

[6] M. Madson, C. Dunand, X. Li, R. Verma, G.F. Vanzin, J. Caplan, D.A. Shoue, N.C. Carpita,W.-D. Reiter, The MUR3 gene of Arabidopsis encodes a xyloglucan galactosyltransferase that is evolutionarily related to animal exostosins, Plant Cell. 15 (2003) 1662-1670.

[7] R.M. Perrin, A.E. DeRocher, M. Bar-Peled, W. Zeng, L. Norambuena, A. Orellana, N.V. Raikhel,K. Keegstra, Xyloglucan fucosyltransferase, an enzyme involved in plant cell wall biosynthesis, Science. 284 (1999) 1976-1979.

[8] S. Vuttipongchaikij, D. Brocklehurst, C. Steele-King, D.A. Ashford, L.D. Gomez,S.J. McQueen-Mason, Arabidopsis GT34 family contains five xyloglucan alpha-1,6-xylosyltransferases, New Phytol. 195 (2012) 585-595.

[9] D.M. Cavalier,K. Keegstra, Two xyloglucan xylosyltransferases catalyze the addition of multiple xylosyl residues to cellohexaose, J Biol Chem. 281 (2006) 34197-34207.

[10] A.T. Culbertson, Y.H. Chou, A.L. Smith, Z.T. Young, A.A. Tietze, S. Cottaz, R. Faure,O.A. Zabotina, Enzymatic Activity of Xyloglucan Xylosyltransferase 5, Plant Physiol. 171 (2016) 1893-1904.

[11] L. von Schantz, F. Gullfot, S. Scheer, L. Filonova, L. Cicortas Gunnarsson, J.E. Flint, G. Daniel, E. Nordberg-Karlsson, H. Brumer,M. Ohlin, Affinity maturation generates greatly improved xyloglucan-specific carbohydrate binding modules, BMC Biotechnol. 9 (2009) 92.

[12] G.F. Vanzin, M. Madson, N.C. Carpita, N.V. Raikhel, K. Keegstra,W.D. Reiter, The mur2 mutant of Arabidopsis thaliana lacks fucosylated xyloglucan because of a lesion in fucosyltransferase AtFUT1, Proc Natl Acad Sci U S A. 99 (2002) 3340-3345.

[13] D.M. VeytsmanCavalier, O. Lerouxel, L. Neumetzler, K. Yamauchi, A. Reinecke, G. Freshour, O.A. Zabotina, M.G. Hahn, I. Burgert, M. Pauly, N.V. Raikhel,K. Keegstra, Disrupting two Arabidopsis thaliana xylosyltransferase 
genes results in plants deficient in xyloglucan, a major primary cell wall component, Plant Cell. 20 (2008) 1519-1537.

[14] C. Xiao, T. Zhang, Y. Zheng, D.J. Cosgrove,C.T. Anderson, Xyloglucan Deficiency Disrupts Microtubule Stability and Cellulose Biosynthesis in Arabidopsis, Altering Cell Growth and Morphogenesis, Plant Physiology and Biochemistry. 170 (2016) 234-249.

[15] O.A. Zabotina, W.T. van de Ven, G. Freshour, G. Drakakaki, D. Cavalier, G. Mouille, M.G. Hahn, K. Keegstra,N.V. Raikhel, Arabidopsis XXT5 gene encodes a putative alpha-1,6-xylosyltransferase that is involved in xyloglucan biosynthesis, Plant J. 56 (2008) 101-115.

[16] Y.B. Park,D.J. Cosgrove, Changes in cell wall biomechanical properties in the xyloglucan-deficient $x x t 1 / x x t 2$ mutant of Arabidopsis, Plant Physiol. 158 (2012) 465-475.

[17] K. KAM1CTTamura, T. Shimada, M. Kondo, M. Nishimura,I. Hara-Nishimura, KATAMARI1/MURUS3 Is a novel Golgi membrane protein that is required for endomembrane organization in Arabidopsis, Plant Cell. 17 (2005) 1764-1776.

[18] W.D. Reiter, C. Chapple,C.R. Somerville, Mutants of Arabidopsis thaliana with altered cell wall polysaccharide composition, Plant J. 12 (1997) 335-345.

[19] Y. Kong, M.J. Peña, L. Renna, U. Avci, S. Pattathil, S.T. Tuomivaara, X. Li, W.-D. Reiter, F. Brandizzi, M.G. Hahn, A.G. Darvill, W.S. York,M.A. O’Neill, Galactose-Depleted Xyloglucan is Dysfunctional and Leads to Dwarfism in Arabidopsis, Plant Physiology. 167 (2015) 1296-1306.

[20] Z.J. Gong, Y.Q. Wu, J. Miao, Y. Duan, Y.L. Jiang,T. Li, Global transcriptome analysis of orange wheat blossom midge, Sitodiplosis mosellana (Gehin) (Diptera: Cecidomyiidae) to identify candidate transcripts regulating diapause, PLoS One. 8 (2013) e71564.

[21] J.D. Tedman-Jones, R. Lei, F. Jay, G. Fabro, X. Li, W.D. Reiter, C. Brearley,J.D. Jones, Characterization of Arabidopsis mur3 mutations that result in constitutive activation of defence in petioles, but not leaves, Plant J. 56 (2008) 691-703.

[22] Y.B. Park,D.J. Cosgrove, A revised architecture of primary cell walls based on biomechanical changes induced by substrate-specific endoglucanases, Plant Physiol. 158 (2012) 1933-43.

[23] S. Wolf, K. Hematy,H. Hofte, Growth control and cell wall signaling in plants, Annu Rev Plant Biol. 63 (2012) 381-407.

[24] K. H'maty, P.-E. Sado, A. Van Tuinen, S. Rochange, T. Desnos, S. Balzergue, S. Pelletier, J.-P. Renou,H. Hofte, A receptor-like kinase mediates the response of Arabidopsis cells to the inhibition of cellulose synthesis, Current Biology. 17 (2007) 922-931.

[25] B.D. Kohorn,S.L. Kohorn, The cell wall-associated kinases, WAKs, as pectin receptors, Front Plant Sci. 3 (2012) 1-5.

[26] S. Wolf, D. van der Does, F. Ladwig, C. Sticht, A. Kolbeck, A.K. Schurholz, S. Augustin, N. Keinath, T. Rausch, S. Greiner, K. Schumacher, K. Harter, C. Zipfel,H. Hofte, A receptor-like protein mediates the response to pectin modification by activating brassinosteroid signaling, Proc Natl Acad Sci U S A. 111 (2014) 15261-15266.

[27] M.D. Abramoff, M.P. J.,R.S. J., Image processing with ImageJ, Biophotonics International. 11 (2004) 36-42. 
[28] C. Trapnell, B.A. Williams, G. Pertea, A. Mortazavi, G. Kwan, M.J. van Baren, S.L. Salzberg, B.J. Wold,L. Pachter, Transcript assembly and quantification by RNA-Seq reveals unannotated transcripts and isoform switching during cell differentiation, Nature Biotechnology. 28 (2010) 511-515.

[29] S. Anders, HTSeq: Analysing high-throughput sequencing data with Python, URL http://www-huber. embl. de/users/anders/HTSeq/doc/overview. html. (2010).

[30] A. Mortazavi, B.A. Williams, K. McCue, L. Schaeffer,B. Wold, Mapping and quantifying mammalian transcriptomes by RNA-Seq, Nature Methods. 5 (2008) 621-628.

[31] M.-A. Dillies, A. Rau, J. Aubert, C. Hennequet-Antier, M. Jeanmougin, N. Servant, C. Keime, G. Marot, D. Castel,J. Estelle, A comprehensive evaluation of normalization methods for Illumina high-throughput RNA sequencing data analysis, Briefings in Bioinformatics. 14 (2013) 671-683.

[32] L. Wang, Z. Feng, X. Wang, X. Wang,X. Zhang, DEGseq: an R package for identifying differentially expressed genes from RNA-seq data, Bioinformatics. 26 (2010) 136-138.

[33] Y. Benjamini,Y. Hochberg, Controlling the false discovery rate: a practical and powerful approach to multiple testing, Journal of the Royal Statistical Society. Series B (Methodological). (1995) 289-300.

[34] Z. Du, X. Zhou, Y. Ling, Z. Zhang,Z. Su, agriGO: a GO analysis toolkit for the agricultural community, Nucleic Acids Research. (2010) gkq310.

[35] C. Xie, X. Mao, J. Huang, Y. Ding, J. Wu, S. Dong, L. Kong, G. Gao, C.-Y. Li,L. Wei, KOBAS 2.0: a web server for annotation and identification of enriched pathways and diseases, Nucleic Acids Research. 39 (2011) 316-322.

[36] O. Thimm, O. Blasing, Y. Gibon, A. Nagel, S. Meyer, P. Kruger, J. Selbig, L.A. Muller, S.Y. Rhee,M. Stitt, MAPMAN: a user-driven tool to display genomics data sets onto diagrams of metabolic pathways and other biological processes, Plant Journal. 37 (2004) 914-939.

[37] N. Mitsuda,M. Ohme-Takagi, Functional analysis of transcription factors in Arabidopsis, Plant Cell Physiol. 50 (2009) 1232-1248.

[38] B. Zhang,S. Horvath, A general framework for weighted gene co-expression network analysis, Stat Appl Genet Mol Biol. 4 (2005) 1544-6115.

[39] R. Saito, M.E. Smoot, K. Ono, J. Ruscheinski, P.-L. Wang, S. Lotia, A.R. Pico, G.D. Bader,T. Ideker, A travel guide to Cytoscape plugins, Nature Methods. 9 (2012) 1069-1076.

[40] S.J. Clough,A.F. Bent, Floral dip: a simplified method forAgrobacterium-mediated transformation of Arabidopsis thaliana, Plant Journal. 16 (1998) 735-743.

[41] K.J. Livak,T.D. Schmittgen, Analysis of relative gene expression data using real-time quantitative PCR and the 2(-Delta Delta CT) Method, Methods. 25 (2001) 402-408.

[42] Y.B. Park,D.J. Cosgrove, Xyloglucan and its interactions with other components of the growing cell wall, Plant Cell Physiol. 56 (2015) 180-94.

[43] E. Jamet, D. Roujol, H. San-Clemente, M. Irshad, L. Soubigou-Taconnat, J.-P. Renou,R. Pont-Lezica, Cell wall biogenesis of Arabidopsis thaliana elongating cells: transcriptomics complements proteomics, BMC Genomics. 10 (2009) 1-12. 
[44] P. Derbyshire, K. Findlay, M.C. McCann,K. Roberts, Cell elongation in Arabidopsis hypocotyls involves dynamic changes in cell wall thickness, Journal of Experimental Botany. 58 (2007) 2079-2089.

[45] M. Irshad, H. Canut, G. Borderies, R. Pont-Lezica,E. Jamet, A new picture of cell wall protein dynamics in elongating cells of Arabidopsis thaliana: confirmed actors and newcomers, BMC Plant Biology. 8 (2008) 94-110.

[46] W.D. Teale, I.A. Paponov,K. Palme, Auxin in action: signalling, transport and the control of plant growth and development, Nat Rev Mol Cell Biol. 7 (2006) 847-859.

[47] E.J. Chapman,M. Estelle, Mechanism of auxin-regulated gene expression in plants, Annual Review of Genetics. 43 (2009) 265-285.

[48] S. Wolf, J. Mravec, S. Greiner, G. Mouille,H. Hofte, Plant cell wall homeostasis is mediated by brassinosteroid feedback signaling, Curr Biol. 22 (2012) 1732-1737.

[49] Q. Tian,J. Reed, Control of auxin-regulated root development by the Arabidopsis thaliana SHY2/IAA3 gene, Development. 126 (1999) 711-721.

[50] H. Leyser, F.B. Pickett, S. Dharmasiri,M. Estelle, Mutations in the AXR3 gene of Arabidopsis result in altered auxin response including ectopic expression from the SAUR-AC1 promoter, Plant Journal. 10 (1996) 403-413.

[51] B.C. Kim, M.S. Soh, B.J. Kang, M. Furuya,H.G. Nam, Two dominant photomorphogenic mutations of Arabidopsis thaliana identified as suppressor mutations of hy2, Plant Journal. 9 (1996) 441-456.

[52] P.J. Overvoorde, Y. Okushima, J.M. Alonso, A. Chan, C. Chang, J.R. Ecker, B. Hughes, A. Liu, C. Onodera,H. Quach, Functional genomic analysis of the AUXIN/INDOLE-3-ACETIC ACID gene family members in Arabidopsis thaliana, Plant Cell. 17 (2005) 3282-3300.

[53] S.D. Clouse,J.M. Sasse, BRASSINOSTEROIDS: Essential Regulators of Plant Growth and Development, Annu Rev Plant Physiol Plant Mol Biol. 49 (1998) 427-451.

[54] Y. Sun, X.Y. Fan, D.M. Cao, W. Tang, K. He, J.Y. Zhu, J.X. He, M.Y. Bai, S. Zhu, E. Oh, S. Patil, T.W. Kim, H. Ji, W.H. Wong, S.Y. Rhee,Z.Y. Wang, Integration of brassinosteroid signal transduction with the transcription network for plant growth regulation in Arabidopsis, Dev Cell. 19 (2010) 765-777.

[55] G. Vert, J.L. Nemhauser, N. Geldner, F. Hong,J. Chory, Molecular mechanisms of steroid hormone signaling in plants, Annu. Rev. Cell Dev. Biol. 21 (2005) 177-201.

[56] T. Takahashi, A. Gasch, N. Nishizawa,N.-H. Chua, The DIMINUTO gene of Arabidopsis is involved in regulating cell elongation, Genes Development. 9 (1995) 97-107.

[57] J. Chory, P. Nagpal,C.A. Peto, Phenotypic and genetic analysis of $\operatorname{det} 2$, a new mutant that affects light-regulated seedling development in Arabidopsis, Plant Cell. 3 (1991) 445-459.

[58] K.A. Feldmann, M.D. Marks, M.L. Christianson,R.S. Quatrano, A Dwarf Mutant of Arabidopsis Generated by T-DNA Insertion Mutagenesis, Science. 243 (1989) 1351-1354.

[59] A. Kauschmann, A. Jessop, C. Koncz, M. Szekeres, L. Willmitzer,T. Altmann, Genetic evidence for an essential role of brassinosteroids in plant development, Plant Journal. 9 (1996) 701-713. 
[60] K. Hematy,H. Hofte, Novel receptor kinases involved in growth regulation, Curr Opin Plant Biol. 11 (2008) 321-8.

[61] B.D. Kohorn, WAKs; cell wall associated kinases, Current Opinion in Cell Biology. 13 (2001) 529-533.

[62] P.M. Ray, P.B. Green,R. Cleland, Role of turgor in plant cell growth. (1972).

[63] G. Guerriero, J.-F. Hausman,G. Cai, No stress! Relax! Mechanisms governing growth and shape in plant cells, Int JMol Sci. 15 (2014) 5094-5114.

[64] D.J. Cosgrove, Growth of the plant cell wall, Nature Rev Mol Cell Biol. 6 (2005) 850-861.

[65] U.G. Hacke,J. Laur, Aquaporins: Channels for the Molecule of Life, Encylopedia Life Science. (2016) 1-6.

[66] R. Aharon, Y. Shahak, S. Wininger, R. Bendov, Y. Kapulnik,G. Galili, Overexpression of a plasma membrane aquaporin in transgenic tobacco improves plant vigor under favorable growth conditions but not under drought or salt stress, Plant Cell. 15 (2003) 439-447.

[67] B. Péret, G. Li, J. Zhao, L.R. Band, U. Voß, O. Postaire, D.-T. Luu, O. Da Ines, I. Casimiro,M. Lucas, Auxin regulates aquaporin function to facilitate lateral root emergence, Nature Cell Biology. 14 (2012) 991-998.

[68] D. Bellincampi, F. Cervone,V. Lionetti, Plant cell wall dynamics and wall-related susceptibility in plant-pathogen interactions, Front Plant Sci. 5 (2014) 1-8.

[69] V. Lionetti, F. Cervone,D. Bellincampi, Methyl esterification of pectin plays a role during plant-pathogen interactions and affects plant resistance to diseases, J Plant Physiol. 169 (2012) 1623-30.

[70] S. Ferrari, D.V. Savatin, F. Sicilia, G. Gramegna, F. Cervone,G.D. Lorenzo, Oligogalacturonides: plant damage-associated molecular patterns and regulators of growth and development, Front Plant Sci. 4 (2013) 49.

[71] G. Pogorelko, V. Lionetti, D. Bellincampi,O. Zabotina, Cell wall integrity: targeted post-synthetic modifications to reveal its role in plant growth and defense against pathogens, Plant Signal Behav. 8 (2013) 1-8.

[72] G. Bethke, A. Thao, G. Xiong, B. Li, N.E. Soltis, N. Hatsugai, R.A. Hillmer, F. Katagiri, D.J. Kliebenstein, M. Pauly,J. Glazebrook, Pectin Biosynthesis Is Critical for Cell Wall Integrity and Immunity in Arabidopsis thaliana, Plant Cell. 28 (2016) 537-556.

[73] V. Lionetti, F. Cervone,D. Bellincampi, Methyl esterification of pectin plays a role during plant-pathogen interactions and affects plant resistance to diseases, J Plant Physiol. 169 (2012) 1623-1630.

[74] V. Lionetti, A. Raiola, L. Camardella, A. Giovane, N. Obel, M. Pauly, F. Favaron, F. Cervone,D. Bellincampi, Overexpression of pectin methylesterase inhibitors in Arabidopsis restricts fungal infection by Botrytis cinerea, Plant Physiol. 143 (2007) 1871-1880.

[75] C. Volpi, M. Janni, V. Lionetti, D. Bellincampi, F. Favaron,R. D'Ovidio, The ectopic expression of a pectin methyl esterase inhibitor increases pectin methyl esterification and limits fungal diseases in wheat, Mol Plant Microbe Interact. 24 (2011) 1012-1019. 
Figure legends

Fig. 1: Etiolated hypocotyl length of WT, xxt2mur3-3, xxt5mur3-3, xxt1xxt2mur3-3, 35Spro:XLT2:mur3-3 and mur3-3. Hypocotyls from at least ten seedlings of each of the six plant lines were measured. Data shown are average $+/-$ SD. (Student's two-tailed t-test with mur3-3; ** $\mathrm{P}<0.01$ ).

Fig. 2: The distribution of gene expression levels. The $X$ axis represents the different plant lines and the $\mathrm{Y}$ axis is the $\log _{10}(\mathrm{RPKM}+1)$ gene expression category $(>0$ and $<5$ ). Most of the genes are expressed at low levels. Only a small number of genes are highly expressed. All six libraries have similar expression patterns.

Fig. 3: The number of differently expressed genes in mur3-3 compared with WT, xxt2mur3-3, xxt5mur3-3, xxt1xxt2mur3-3 and 35Spro:XLT2:mur3-3. A: Up-regulated DEGs in WT, xxt2mur3-3, xxt5mur3-3, xxt1xxt2mur3-3 and 35Spro:XLT2:mur3-3 compared with mur3-3. B: Down-regulated DEGs in WT, xxt2mur3-3, xxt5mur3-3, xxt1xxt2mur3-3 and 35Spro:XLT2:mur3-3 compared with mur3-3.

Fig. 4: Hierarchical cluster analysis of gene expression based on log ratio RPKM data $\left(\log _{10}(\mathbf{R P K M}+1)\right)$. Green represents the lowest expression and red represents highest expression. Each column represents one of the six plant lines. Rows represent transcriptional units.

Fig. 5: Functional categories of DEGs in the Gene Ontology. The top four of the most significantly enriched ( $p$-value <0.05) GO terms of biological process, molecular function and cellular component were analyzed in the comparisons of mur3-3 with WT, $x x t 2 m u r 3-3, x x t 5 m u r 3-3$ and $x x t 1 x x t 2 m u r 3-3$.

Fig. 6: DEGs showed in Venn diagram form. A: Venn diagram showing the numbers of overlapped DEGs detected in comparisons of mur3-3 with WT, $x x t 2 m u r 3-3, x x t 5 m u r 3-3, x x t 1 x x t 2 m u r 3-3$ and 35Spro:XLT2:mur3-3. B: Venn diagram showing the numbers of overlapped DEGs detected in comparisons of mur3-3 with WT, xxt2mur3-3, xxt5mur3-3 and xxt1xxt2mur3-3. The Venn diagrams include both up- and down-regulated genes.

Fig. 7: Overview of 550 overlapped DEGs involved in different metabolic and regulatory processes visualized by MapMan. A: Overview display of DEGs assigned to metabolism. CHO, carbohydrates; OPP, oxidative pentose phosphate pathway; TCA, tricarboxylic acid cycle. B: Overview display of DEGs assigned to regulatory processes. C: Overview display of DEGs assigned to biotic stress processes. Genes significantly up- and down-regulated in WT and suppressor lines compared with mur3-3 are indicated in red and green, respectively. Scale bars display $\log _{2}$-fold changes.

Fig. 8: Overexpression of selected genes rescues the short hypocotyl phenotype of the mur3-3 mutant. A: The hypocotyl phenotypes of mur3-3 lines over expressing XTH4, XTH30, PME3, EXPA11, ROT3, IAA6, IAA17, WAG2, AT5G37790, MYB88 or TIP2;3. B: Hypocotyl length of WT, mur3-3 and transgenic lines. Between 10 and 15 hypocotyls were measured for each transgenic line. Data shown are average +/- SD. (Student's two-tailed t-test with mur3-3; ** $\mathrm{P}<0.01$ ). 
Fig. 9: The weighted co-expression network of differentially expressed genes including cell wall-related genes, TFs, hormone genes, protein kinase genes, aquaporins and chlorophyll a-b binding protein genes. The different color represents different types of genes and the size of the circle represents the quantity of connection joints with other genes.

Fig. 10: Comparison of the changes in the abundance and expression levels of selected genes. A: The relative expression values representing $\log _{2}$ (fold change) among comparisons of mur3-3 with WT and four suppressor lines. RPKM values were used in DGE-seq and $2^{-\triangle \Delta C T}$ values were used in qRT-PCR for fold-change calculation. B: Coefficient analysis between gene expression ratios obtained from DGE-seq and qRT-PCR data. 
Table 1 Summary of reads numbers based on DGE-seq of the six plant lines and the number of reads mapped to the genome.

\begin{tabular}{lllllll}
\hline Sample name & Wild type & mur3-3 & xxt2mur3-3 & xxt5mur3-3 & xxt1xxt2mur3-3 & 35Spro:XLT2:mur3-3 \\
\hline Raw reads & 15225291 & 10980105 & 16935349 & 11712348 & 10403746 & 12378356 \\
Clean reads & 14789989 & 10793421 & 16686602 & 11551898 & 10225651 & 12207798 \\
Clean ratio & $97.14 \%$ & $98.30 \%$ & $98.53 \%$ & $98.63 \%$ & $98.29 \%$ & $98.62 \%$ \\
clean bases $(\mathrm{Mb})$ & 740 & 540 & 830 & 580 & 510 & 610 \\
Q20 $(\%)$ & 99.04 & 98.96 & 99.03 & 98.95 & 99.03 & 98.84 \\
GC content $(\%)$ & 45.26 & 45.92 & 45.48 & 45.31 & 45.65 & 45.48 \\
Total mapped reads & 14350871 & 10446763 & 16213637 & 11172751 & 9887405 & 11790956 \\
Mapping ratio & $97.03 \%$ & $96.79 \%$ & $97.17 \%$ & $96.72 \%$ & $96.69 \%$ & $96.59 \%$ \\
\hline
\end{tabular}

Table 2 Cell wall-related DEGs that differed significantly in comparisons of mur3-3 with WT, xxt2mur3-3, xxt5mur3-3, xxt1xxt2mur3-3 and 35Spro:XLT2:mur3-3.

\begin{tabular}{llllllll}
\hline & & \multicolumn{9}{c}{$\log 2$. Fold_change. } & \\
\cline { 3 - 7 } Type & & WT & xxt2mur3-3 & xxt5mur3-3 & xxt1xxt2mur3-3 & 35Spro:XLT2:mur3-3 & Gene Name \\
& Gene ID & vs & vs & vs & vs & vs & mur3-3 \\
\hline Cell wall & mur3-3 & mur3-3 & mur3-3 & mur3-3 & AGP22 \\
& AT5G53250 & 2.76 & 2.63 & 3.03 & 3.00 & 1.70 & AGP24 \\
& AT5G40730 & 2.43 & 1.96 & 2.33 & 2.36 & 1.20 & AGP5 \\
& AT1G35230 & -5.29 & -2.49 & -4.48 & -6.00 & -1.69 & SAG14 \\
& AT5G20230 & -6.93 & -2.02 & -4.82 & -6.74 & -1.73 & BGAL6 \\
& AT5G63800 & 1.81 & 1.47 & 1.52 & 1.47 & 1.01 & IGMT5
\end{tabular}




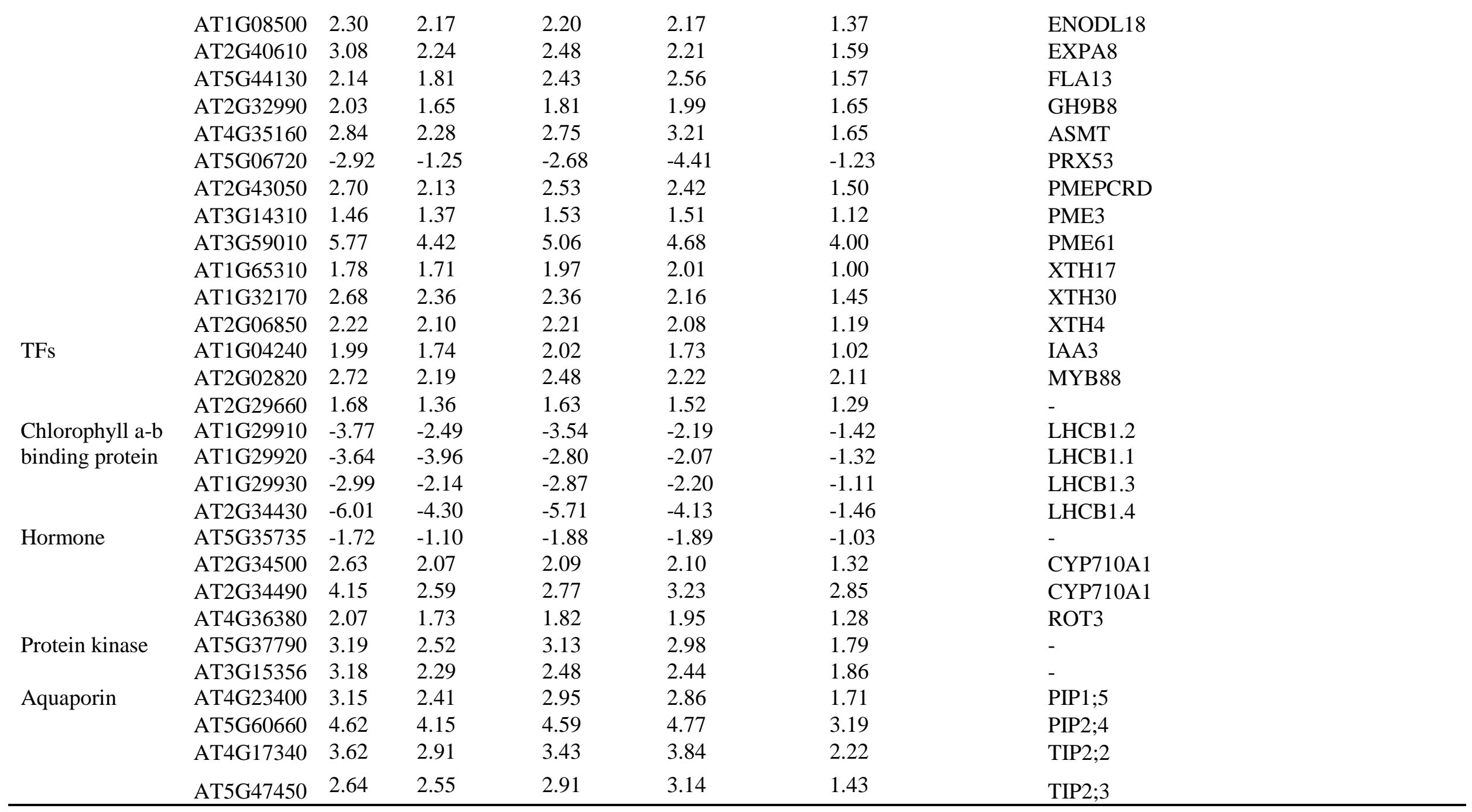




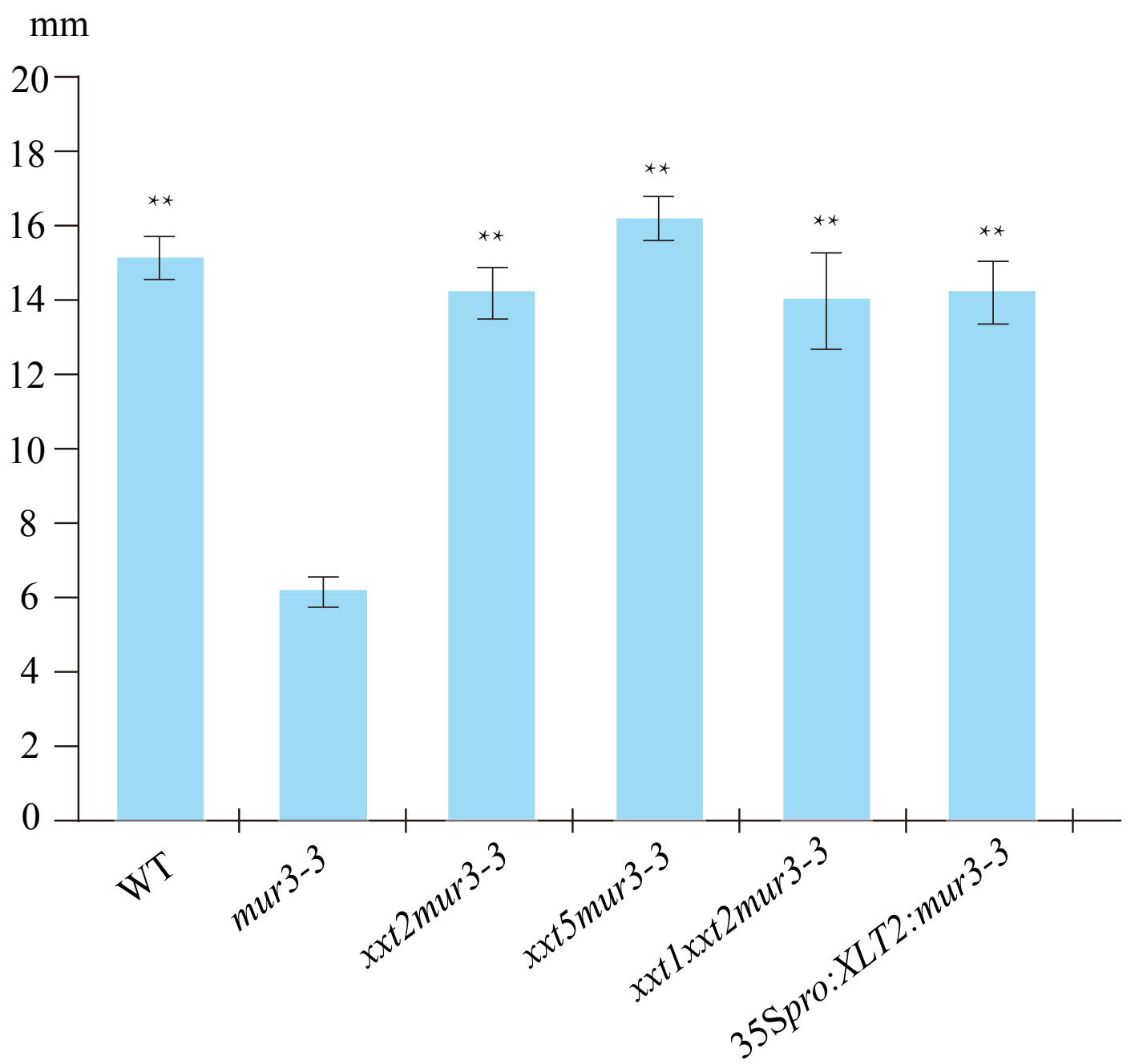




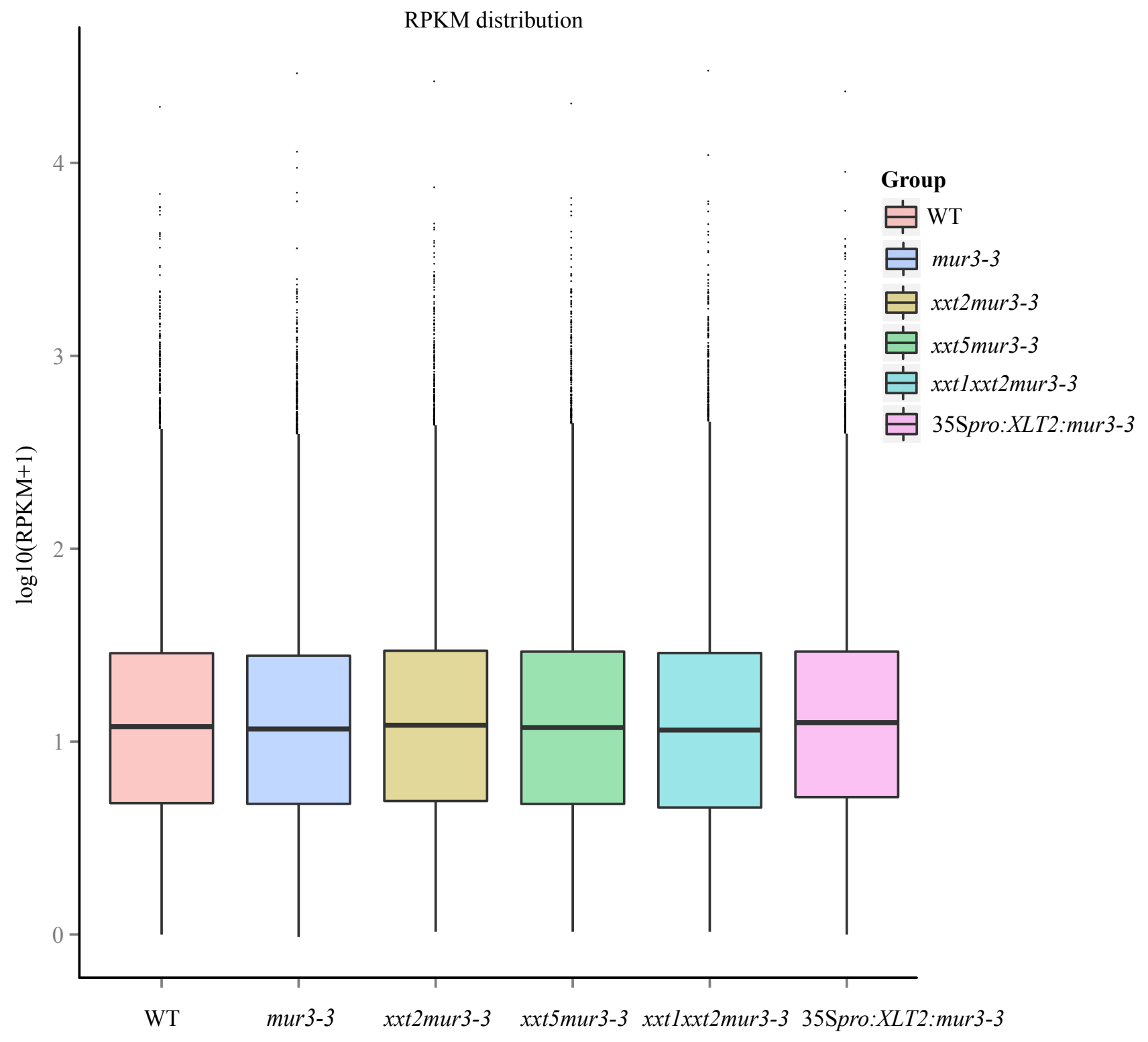



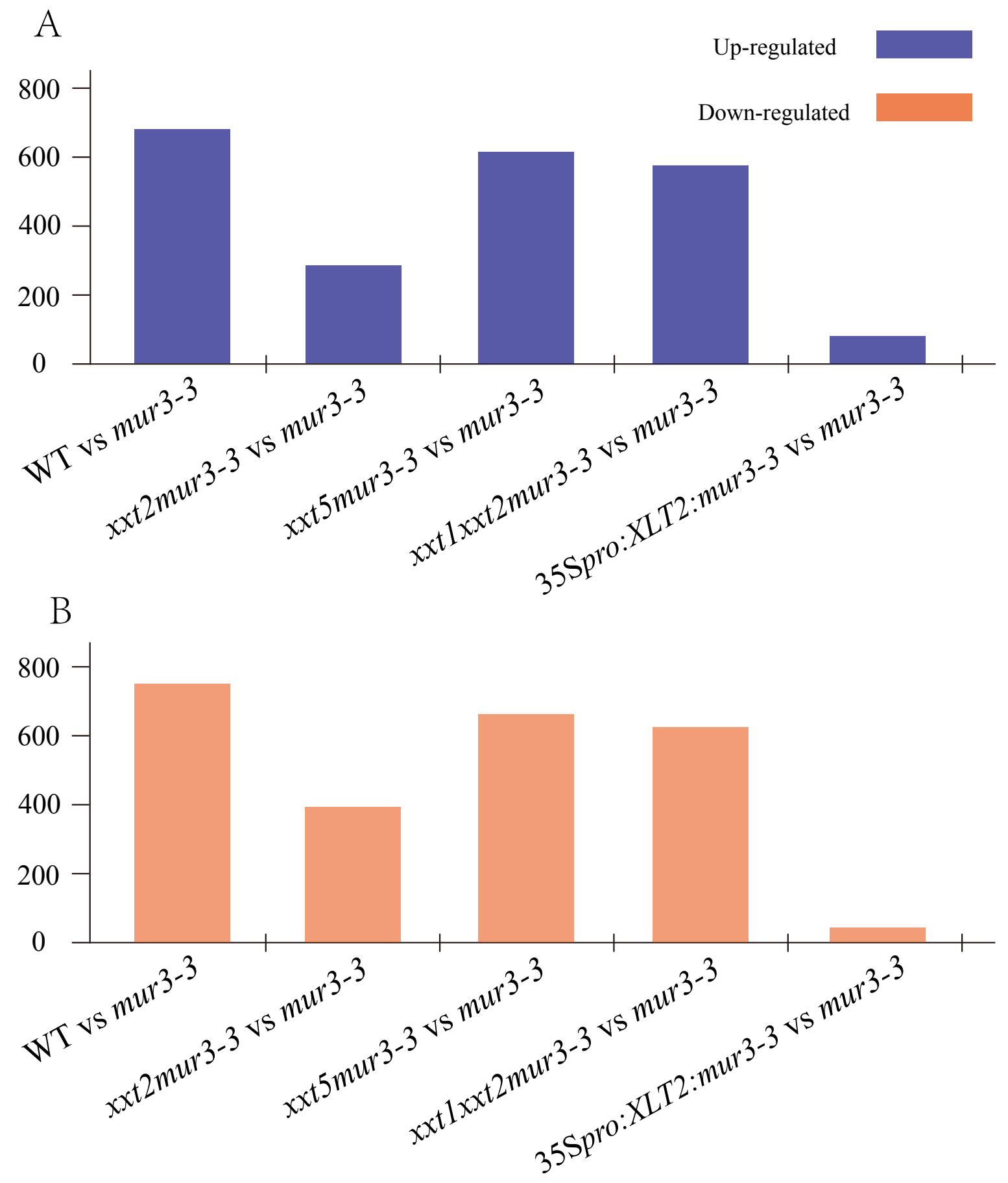


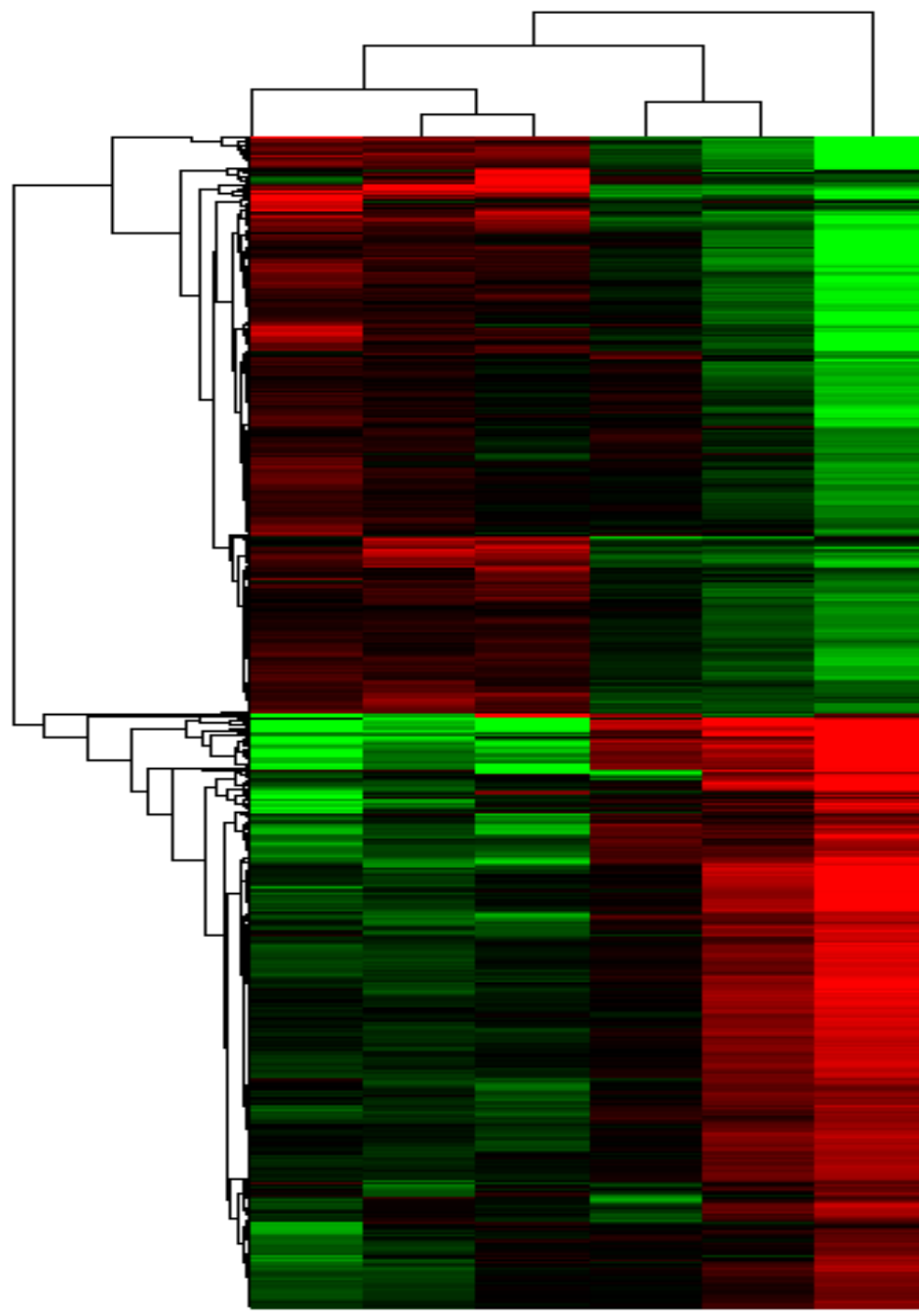

highest

expression

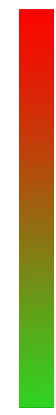

lowest

expression

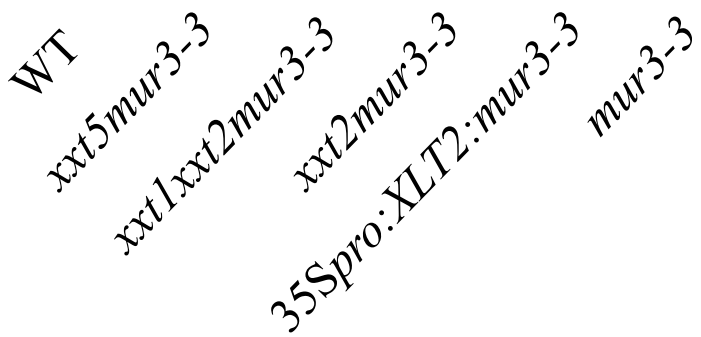




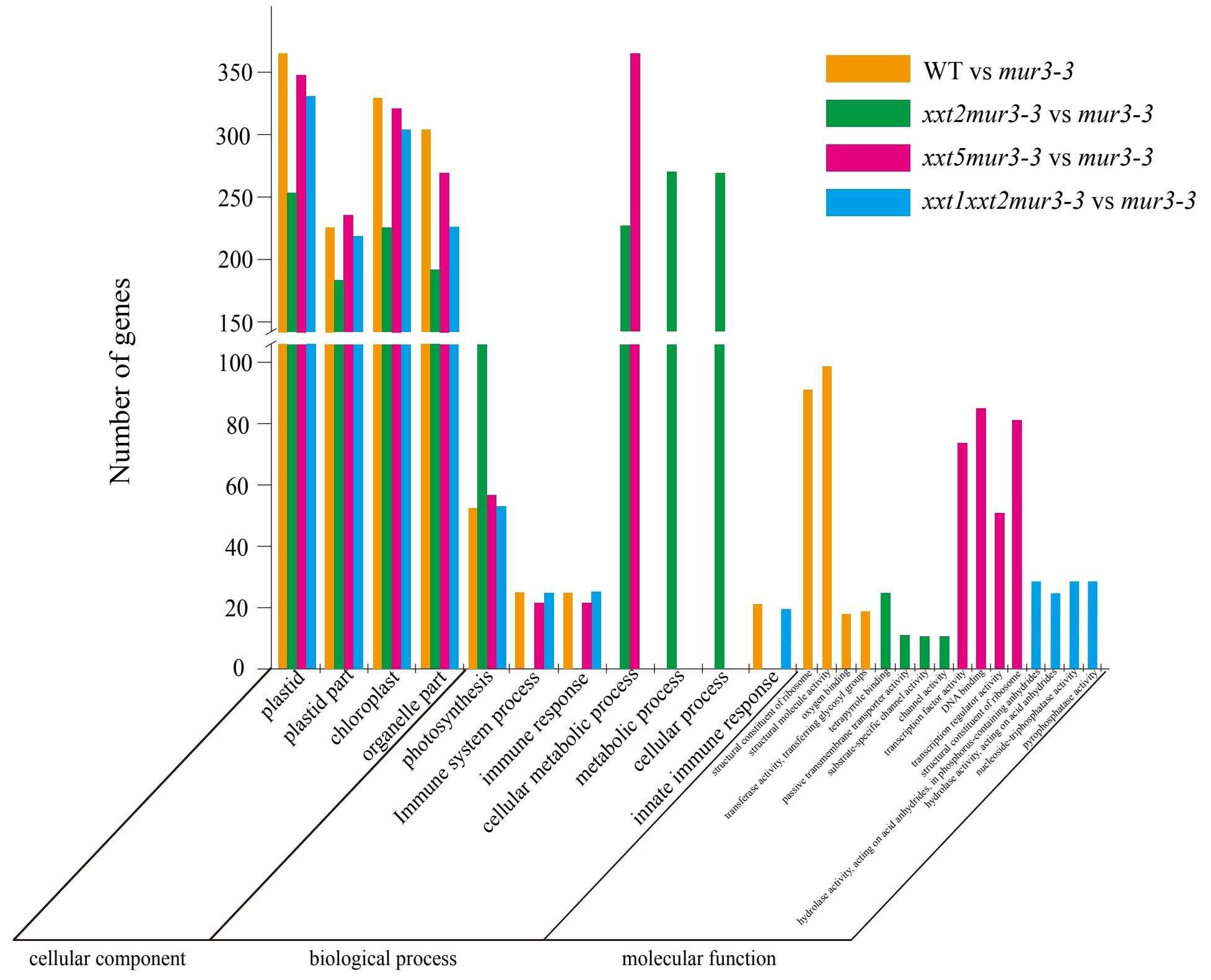


$x x+2 m u r 3-3$ vs mur3-3

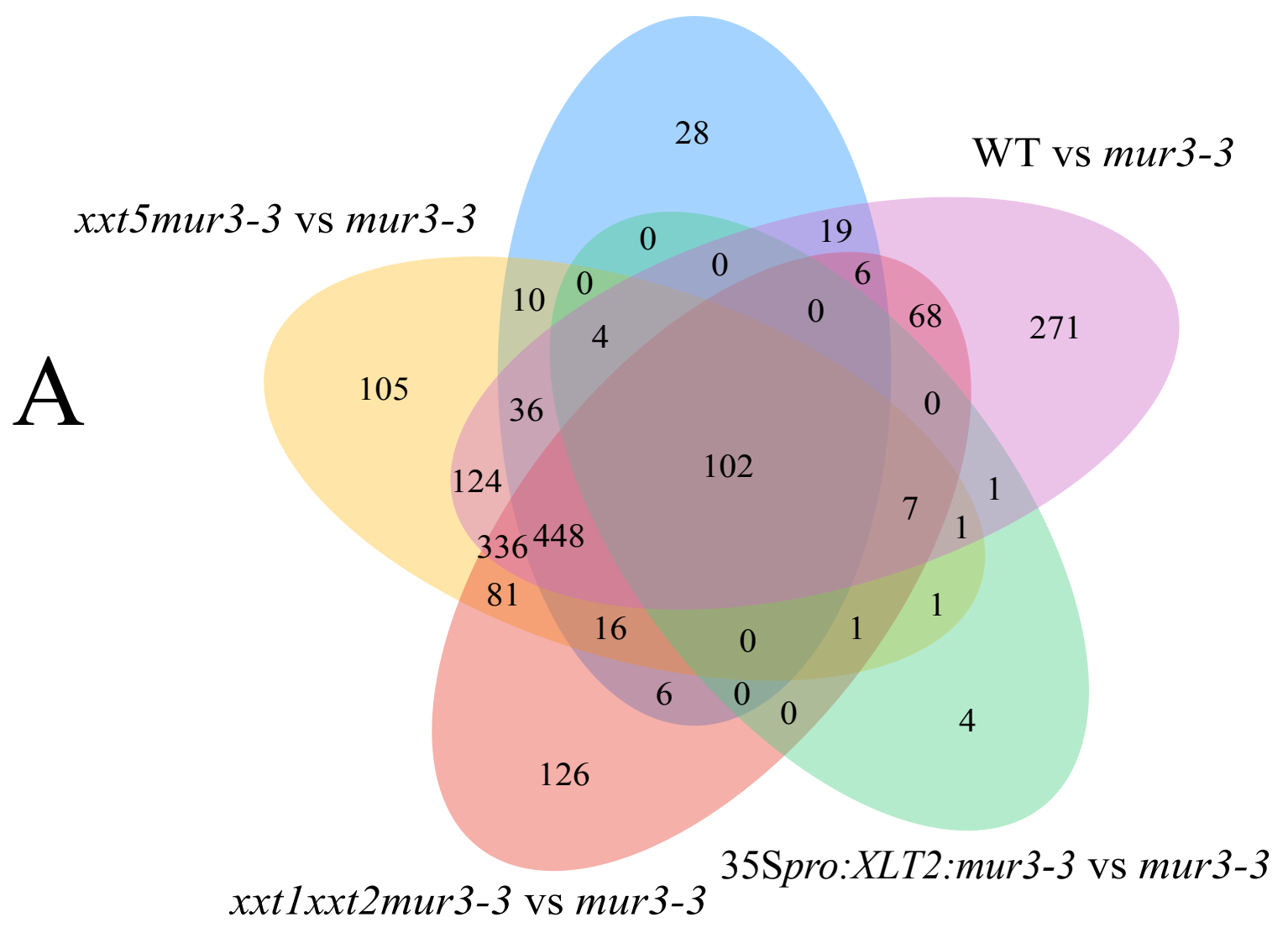

xxt2mur3-3 vs mur3-3 xxt5mur3-3 vs mur3-3

WT vs mur3-3

19

272
28

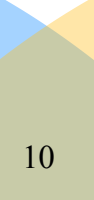

0

125

40 xxt1xxt2mur3-3 vs mur3-3 
A

minor $\mathrm{CHO}$

尽
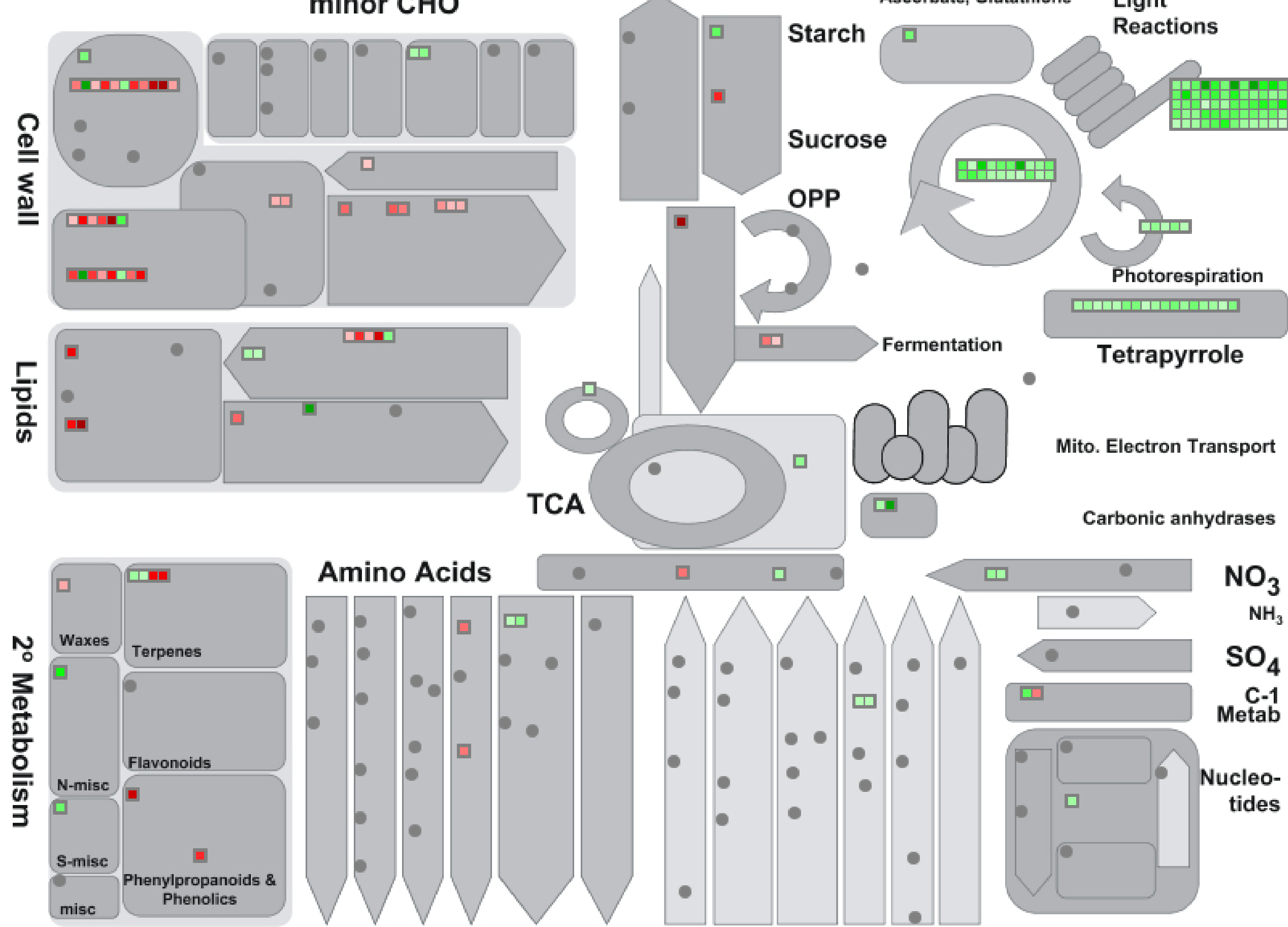

Amino Acids
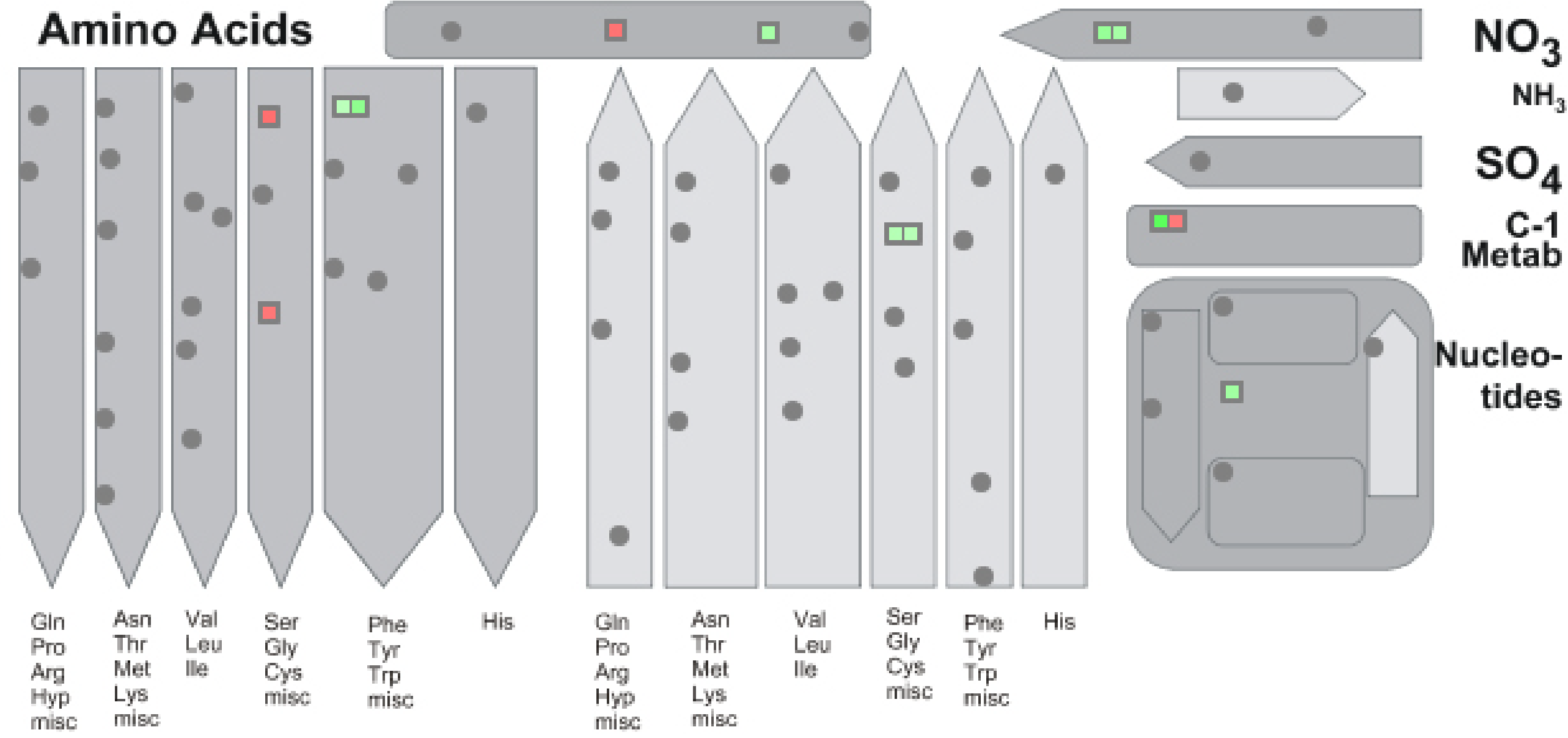

B
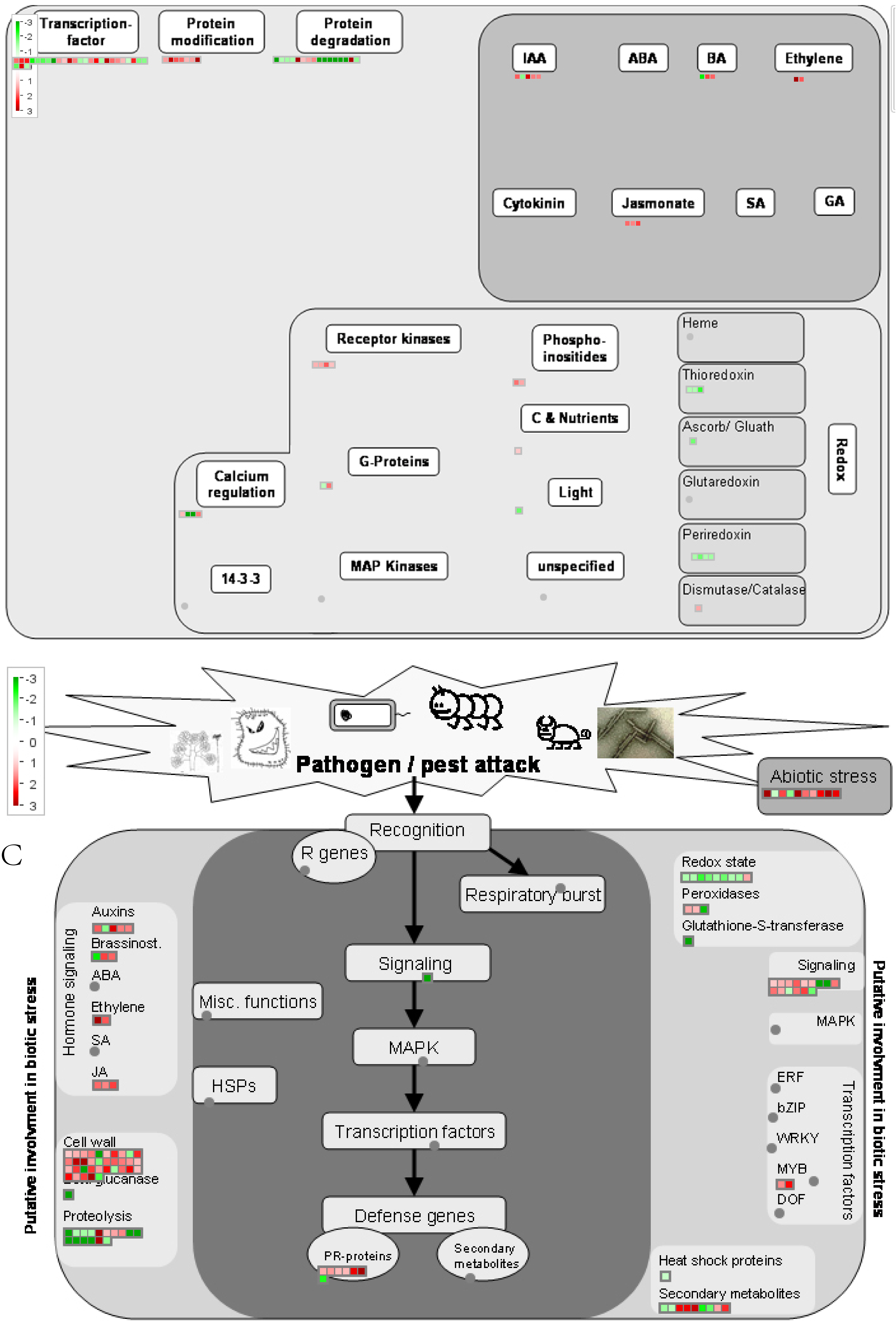
A

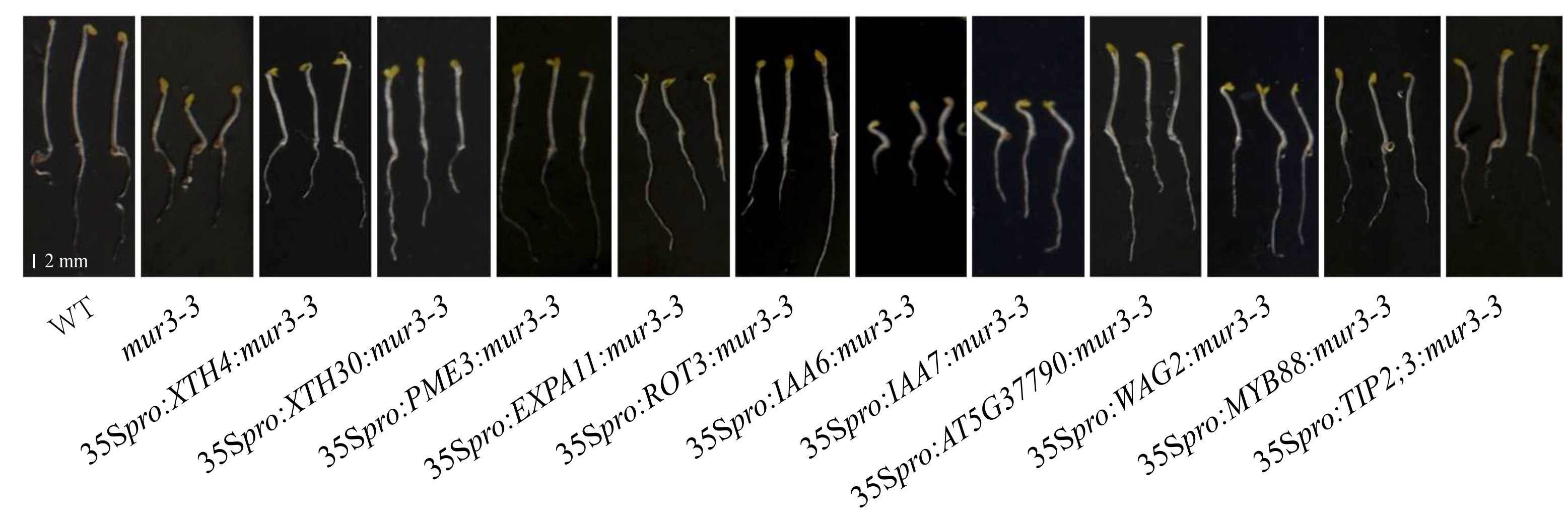

B

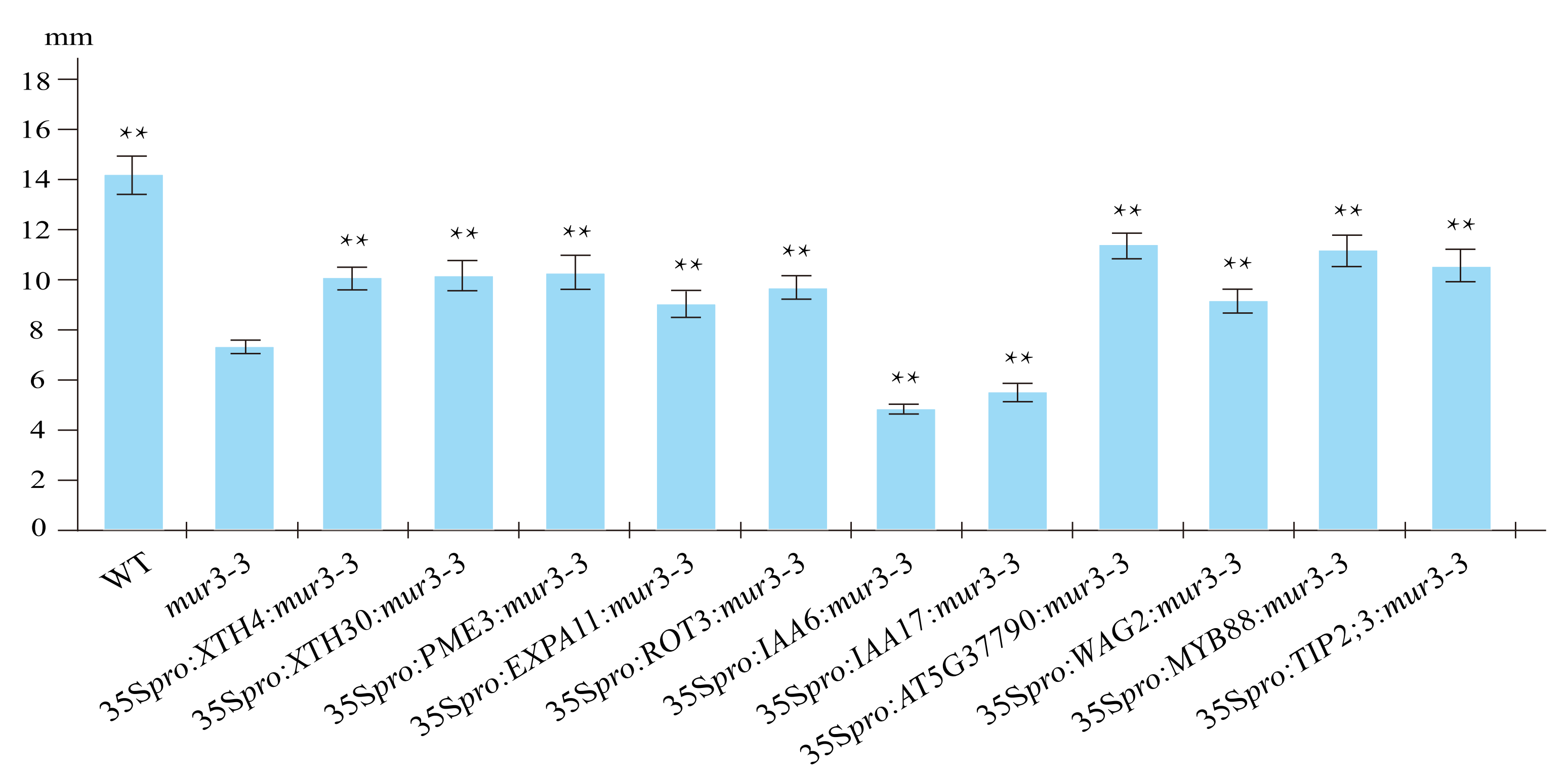




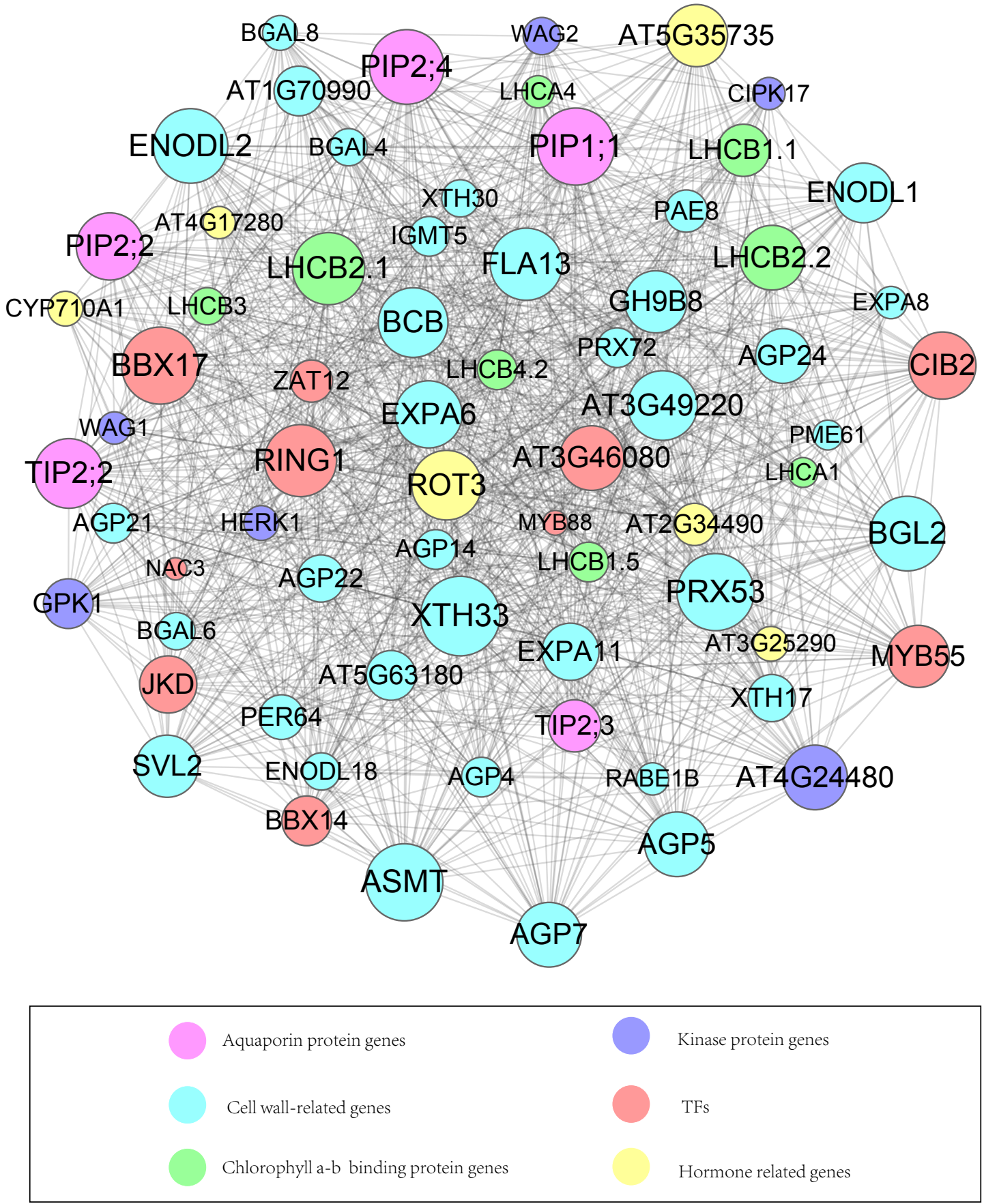



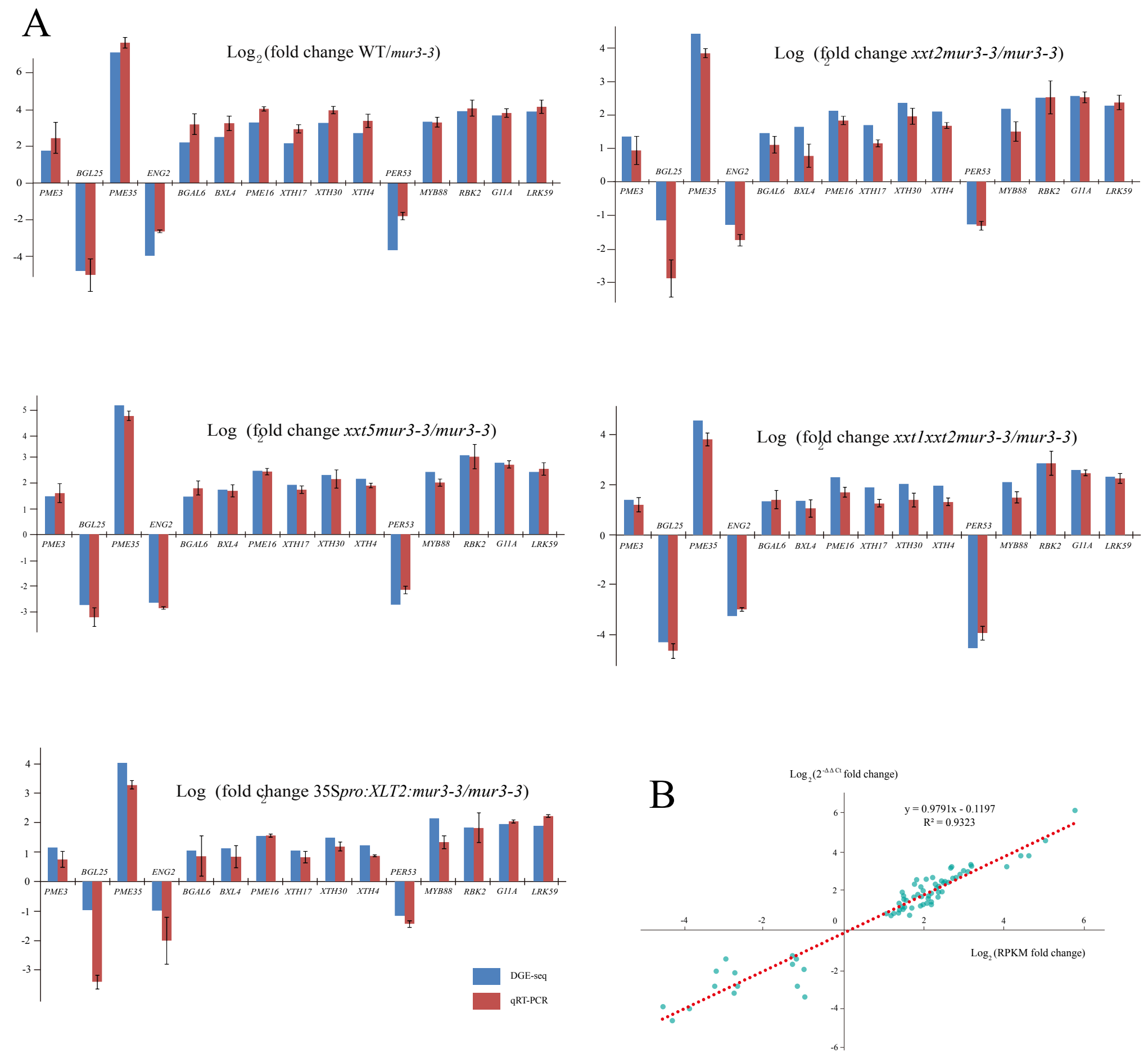